\title{
Reciprocals of Binary Power Series
}

\author{
Joshua N. Cooper* \\ Courant Institute of Mathematics, New York University, New York, NY \\ jcooper@cims.nyu.edu \\ Dennis Eichhorn \\ California State University, East Bay, Hayward, CA \\ eichhorn@mcs.csueastbay.edu \\ Kevin O'Bryant ${ }^{\dagger}$ \\ University of California, San Diego, San Diego, CA \\ City University of New York, College of Staten Island, New York, NY \\ kevin@member.ams.org
}

September 20, 2018

\begin{abstract}
If $A$ is a set of nonnegative integers containing 0 , then there is a unique nonempty set $B$ of nonnegative integers such that every positive integer can be written in the form $a+b$, where $a \in A$ and $b \in B$, in an even number of ways. We compute the natural density of $B$ for several specific sets $A$, including the Prouhet-Thue-Morse sequence, $\{0\} \cup\left\{2^{n}: n \in \mathbb{N}\right\}$, and random sets, and we also study the distribution of densities of $B$ for finite sets $A$. This problem is motivated by Euler's observation that if $A$ is the set of $n$ that have an odd number of partitions, then $B$ is the set of pentagonal numbers $\{n(3 n+1) / 2: n \in \mathbb{Z}\}$. We also elaborate the connection between this problem and the theory of de Bruijn sequences and linear shift registers.
\end{abstract}

\section{Introduction}

There is a unique set $B$ of nonnegative integers with the property that each positive integer can be written in the form $s^{2}+b(s \in \mathbb{N}:=\{0,1,2, \ldots\}, b \in B)$ in an even number of ways. Specifically,

$$
B=\{0,1,2,3,5,7,8,9,13,17,18,23,27,29,31,32,35, \ldots\} .
$$

Are the even numbers in $B$ exactly those of the form $2 k^{2}$ ? Does $B$ have positive density?

Before addressing these two questions, we restate and motivate the problem in greater generality. Given any sets $A, B \subseteq \mathbb{N}$, the asymmetric additive representation function is defined by

$$
R(n):=\#\{(a, b): n=a+b, a \in A, b \in B\} ;
$$

\footnotetext{
*Supported by NSF grant DMS-0303272

†Supported by NSF grant DMS-0202460.
} 


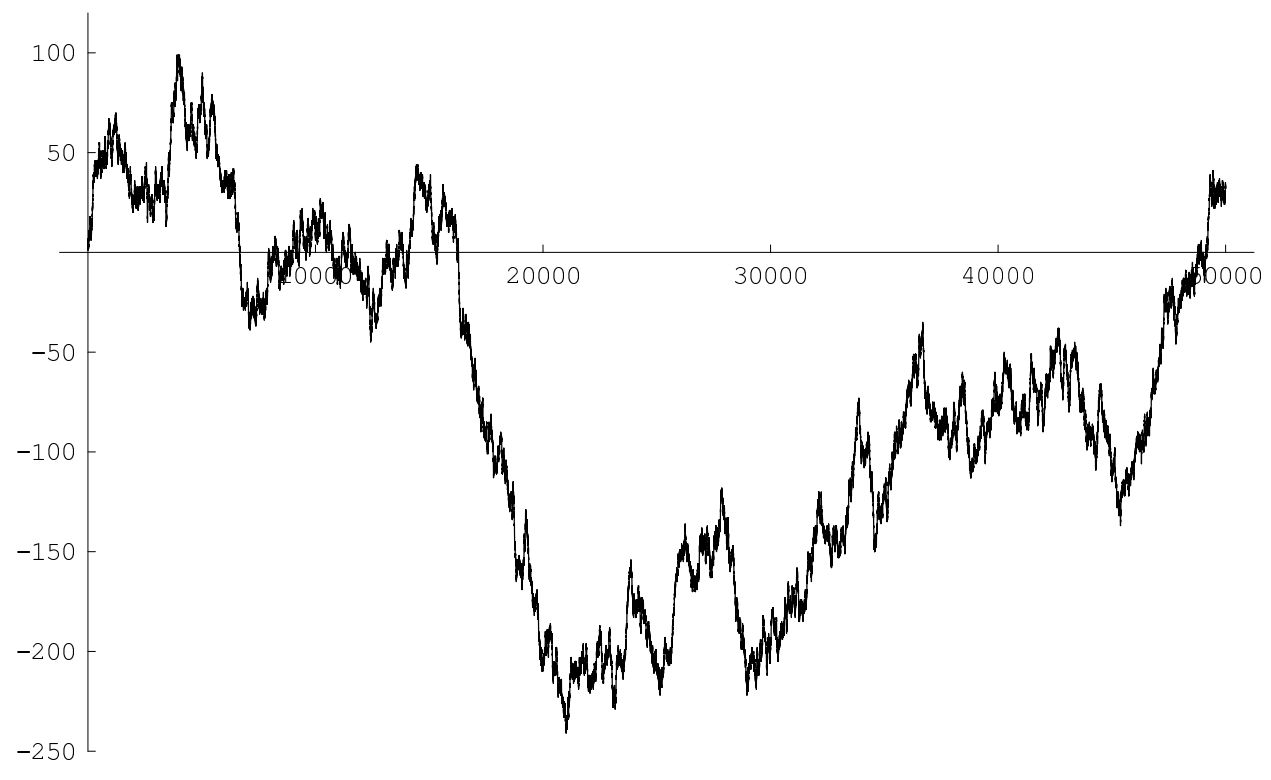

Figure 1: The points $\left(n,\left|P_{\text {odd }} \cap[0, n]\right|-\left|P_{\text {even }} \cap[0, n]\right|-1\right)=\left(n, 2\left|P_{\text {odd }} \cap[0, n]\right|-n\right)$

equivalently, we could define $R$ by noting that

$$
\left(\sum_{a \in A} q^{a}\right)\left(\sum_{b \in B} q^{b}\right)=\sum_{n=0}^{\infty} R(n) q^{n} .
$$

We are interested in the situation where $R(0)=1$ and $R(n) \equiv 0(\bmod 2)$ for $n>0$, i.e., the situation where $\sum_{n} R(n) q^{n}=1$ in the ring of power series $\mathbb{F}_{2}[[q]]$. In this case, we say that $A$ and $B$ are reciprocals, and we write $\bar{A}=B$ and $\bar{B}=A$. The general problem of this paper is to find the reciprocals of several special sets $A$, and to draw some conclusions about "typical" properties of reciprocals. We are particularly concerned with the relative density,

$$
\delta(\bar{A}, n):=\frac{|\bar{A} \cap[0, n]|}{n+1},
$$

and the density $\delta(\bar{A}):=\lim _{n \rightarrow \infty} \delta(\bar{A}, n)$ (when the limit exists).

We began studying this problem after reading two articles by Berndt, Yee, and Zaharescu [5]6], where bounds on the density of the set $P_{\text {odd }}:=\{n \in \mathbb{N}: p(n) \equiv 1(\bmod 2)\}$, with $p(n)$ being the ordinary partition function ${ }^{\mathrm{a}}$, are proved. The starting point for their work is Euler's pentagonal number theorem [12] Theorem 10.9], and in particular that the reciprocal of $P_{\text {odd }}$ is the set $\{n(3 n+$ 1) $/ 2: n \in \mathbb{Z}\}$ of pentagonal numbers. Since the known bounds (see [1] and [1] for the currently-best results) on the thickness of $P_{\text {odd }}$ are so strikingly far from what is believed to be true, we felt that it would be beneficial to study the "reciprocal" notion in a more general setting.

$P_{\text {odd }}$ is pictured in Figure 1 where not only does it appear to have density $1 / 2$, but the walk defined by $w(n):=2\left|P_{\text {odd }} \cap[0, n]\right|-n$ visually appears to be a simple random walk. See [13] for

${ }^{\mathrm{a}} p(n)$ is the number of ways to write $n$ as a sum of nonincreasing positive integers. For example, $4=3+1=$ $2+2=2+1+1=1+1+1+1$, so $p(4)=5$. 
a report of more elaborate statistical tests on the set $P_{\text {odd }}$. We note that while $p(n)$ appears to be uniformly distributed modulo 2 and 3, it has been known since the time of Ramanujan to not be uniformly distributed modulo 5, 7, or 11 .

In contrast to that of the pentagonal numbers, the density of the reciprocal of the squares appears to drop off steadily to 0 . Set $S:=\left\{n^{2}: n \in \mathbb{N}\right\}$, with reciprocal $\bar{S}$. The relative density of $\bar{S}$ is pictured in Figure 2 In Section 6.1 we prove that the even numbers in $\bar{S}$ are precisely $\left\{2 n^{2}: n \in \mathbb{N}\right\}$, and we characterize the $n \in \bar{S}$ with $n \equiv 1(\bmod 4)$ as those $n$ whose prime factorization has a particular shape. Those $n \in \bar{S}$ with $n \equiv 3(\bmod 4)$ are characterized in terms of the number of representations of $n$ by certain quadratic forms.

Generalizing the squares and pentagonal numbers, we treat

$$
\Theta\left(c_{1}, c_{2}\right):=\left\{c_{1} n+c_{2} \frac{n(n-1)}{2}: n \in \mathbb{Z}\right\}
$$

for general $c_{1}$ and $c_{2}$ in Section [6. A few interesting special cases are the binomial coefficients $\Theta(0,1)=\left\{\left(\begin{array}{l}n \\ 2\end{array}\right): n \in \mathbb{N}\right\}$, the squares $\Theta(1,2)$, and the pentagonal numbers $\Theta(1,3)$.

Conjecture 1.1. The reciprocal of the set $\Theta\left(c_{1}, c_{2}\right)$, where $0 \leq 2 c_{1} \leq c_{2}$ and $\operatorname{gcd}\left(c_{1}, c_{2}\right)=1$, has density 0 if $c_{2} \equiv 2(\bmod 4)$, and otherwise has density $1 / 2$. More precisely, if $c_{2} \equiv 2(\bmod 4)$, then

$$
\lim _{n \rightarrow \infty} \frac{\left|\overline{\Theta\left(c_{1}, c_{2}\right)} \cap[0, n]\right|}{n / \log n}=C,
$$

for some positive constant $C$ depending only on $c_{2}$. If $c_{2} \not \equiv 2(\bmod 4)$, then

$$
\limsup _{n \rightarrow \infty}\left|\frac{\left|\overline{\Theta\left(c_{1}, c_{2}\right)} \cap[0, n]\right|-n / 2}{\sqrt{n \log \log (n) / 2}}\right|=1 .
$$

Numerically, it seems that the constant $C$ is 2 if $c_{2}=2$ or 6 , and $C=4$ if $c_{2}=10$. We lack sufficient data to guess the other values. The authors believe that the non-effective $c_{2} \equiv 2(\bmod 4)$ case might be provable by showing that the generating function of $\Theta\left(c_{1}, c_{2}\right)$ is congruent modulo 2 to an integer-weight modular form, which has almost all of its Fourier coefficients even. This is outside the scope of this paper, and we leave it as an area for further study.

The $c_{2} \not \equiv 2(\bmod 4)$ case is motivated by the celebrated law of the iterated logarithm. Let $X_{1}, X_{2}, \ldots$ be independent random variables taking the values 0 and 1 with probability $1 / 2$. The law of the iterated logarithm states that

$$
\limsup _{n \rightarrow \infty}\left|\frac{\sum_{i=1}^{n} X_{i}-n / 2}{\sqrt{n \log \log (n) / 2}}\right|=1
$$

with probability 1 . What we actually would like to conjecture is that reciprocal of $\Theta\left(c_{1}, c_{2}\right)$, with $c_{2} \not \equiv 2(\bmod 4)$, is statistically indistinguishable from a truly random set with density $1 / 2$. The phrase "statistically indistinguishable" is too vague, however, so in Conjecture 1.1 we have settled for this one specific statistic.

The natural expectation is that, barring some cosmic coincidence or obvious structure, the reciprocal of a set should have density $1 / 2$. This is affirmed by the case of a random set, which we handle in detail in Section [3] let $X_{1}, X_{2}, \ldots$ be independent random variables taking the values 0 and 1 , with probabilities bounded away from 0 and 1 , and set $F:=\{0\} \cup\left\{n: X_{n}=1\right\}$. Theorem 3.1 states that the reciprocal of $F$ has density $1 / 2$ with probability 1 . This makes the sets whose reciprocals do not have density $1 / 2$ the interesting ones. 

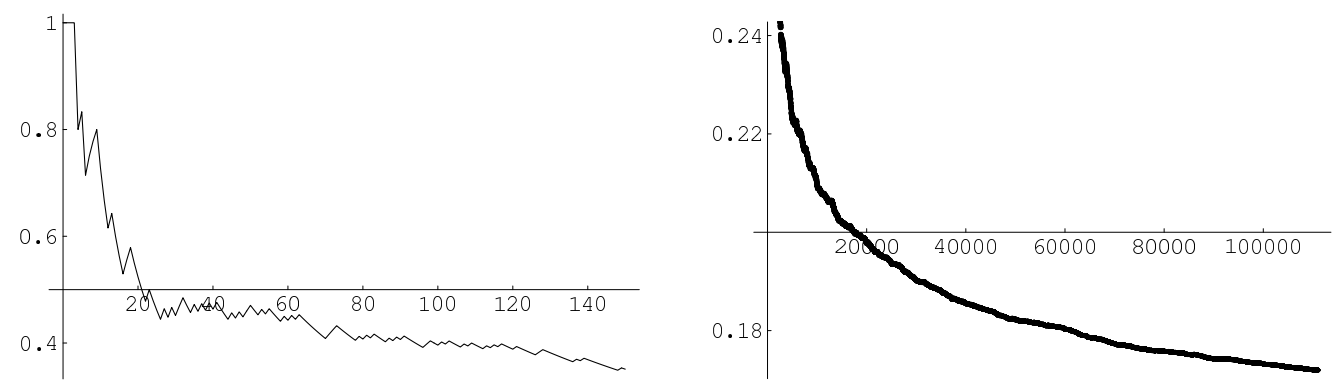

Figure 2: The relative density of the reciprocal of the set of squares

Our purpose is to identify relevant properties of those sets whose reciprocals have density different from $1 / 2$. Specifically, in addition to random sets and $\Theta\left(c_{1}, c_{2}\right)$, we consider finite sets, the set of powers of two, and the set of Prouhet-Thue-Morse numbers.

- Finite sets: the reciprocal has a rational density, and appears to typically have density slightly below $1 / 2$. We identify through algebraic properties two infinite classes of polynomials, one whose reciprocals have density strictly larger than $1 / 2$, and one whose reciprocals have density at most $1 / 2$.

- Powers of 2: the reciprocal of the thin set $\{0\} \cup\left\{2^{n}: n \in \mathbb{N}\right\}$ is the thin set $\left\{2^{n}-1: n \in \mathbb{N}\right\}$. In particular, we describe the reciprocal of $\{0\} \cup\left\{2^{m n}: n \in \mathbb{N}\right\}$ for every $m \in \mathbb{N}$.

- Prouhet-Thue-Morse numbers ${ }^{\mathrm{b}}$ : the reciprocal of

$$
T:=\{n \in \mathbb{N} \text { : the binary expansion of } n \text { contains an even number of " } 1 \text { "s }\} \text {. }
$$

has density $1 / 3$. Specifically, we prove that $k \in \bar{T}$ if and only if $k=0$ or $(k \pm 1) / 4$ is an integer whose binary expansion ends in an even number of zeros.

The strongest conjecture that is consistent with our theorems, our experiments, and Conjecture 1.1 is Conjecture 1.2

Conjecture 1.2. If a set contains 0 , is not periodic, and is uniformly distributed modulo every power of 2, then its reciprocal has positive density.

We now include a section-by-section agenda for the remainder of the paper.

Section 1) Motivate and contextualize reciprocals of sets.

Section 2 Introduce notation and derive general expressions for reciprocals.

Section 3 Consider reciprocals of random sets with positive density.

Section 4. Consider reciprocals of finite sets.

Section 5. Consider the reciprocal of the powers of 2 , and similar sets.

Section 6] Consider the reciprocal of $\Theta\left(c_{1}, c_{2}\right)$, particularly the squares.

Section 7 ; Consider the Prouhet-Thue-Morse sequence.

\footnotetext{
${ }^{\mathrm{b}} T=\{0,3,5,6,9,10,12,15,17,18,20, \ldots\}$
} 


\section{Notation and General Formulas}

Throughout this paper, we let

$$
\mathcal{F}(q)=f_{0}+f_{1} q+f_{2} q^{2}+\cdots \quad \text { and } \quad \overline{\mathcal{F}}(q)=\bar{f}_{0}+\bar{f}_{1} q+\bar{f}_{2} q^{2}+\cdots
$$

be elements of $\mathbb{F}_{2}[[q]]$ that satisfy the equation

$$
\mathcal{F}(q) \overline{\mathcal{F}}(q)=1
$$

In particular, $f_{0}=\bar{f}_{0}=1$. We define the integer sets $F:=\left\{n \geq 0: f_{n}=1\right\}$ and $\bar{F}:=\left\{n \geq 0: \bar{f}_{n}=\right.$ $1\}$.

Note that (2) implies (for all $k \geq 1$ ) that $\mathcal{F}\left(q^{k}\right) \overline{\mathcal{F}}\left(q^{k}\right)=1$ also. This corresponds to noting that multiplying everything in $F$ by $k$ has the effect of multiplying everything in $\bar{F}$ by $k$. With this in mind, we sometimes make the convenient assumption that $\operatorname{gcd} F=1$.

Our next lemma is a fundamental identity in $\mathbb{F}_{2}[[q]]$, and has a number of remarkable consequences. We use it frequently throughout this paper.

Lemma 2.1. The reciprocal of $\mathcal{F}(q)$ is $\mathcal{F}(q) \mathcal{F}\left(q^{2}\right) \mathcal{F}\left(q^{4}\right) \mathcal{F}\left(q^{8}\right) \cdots$. That is,

$$
1=\mathcal{F}(q) \cdot \prod_{k=0}^{\infty} \mathcal{F}\left(q^{2^{k}}\right)
$$

Proof. First, notice that both sides of this equation have constant term equal to 1 . Also notice that for any fixed $n>0$, only finitely many terms of the infinite product affect the coefficient of $q^{n}$. Thus, the coefficient of $q^{n}$ on the right hand side of (3) is also the coefficient of $q^{n}$ in

$$
\mathcal{F}(q) \cdot \prod_{k=0}^{\left\lfloor\log _{2} n\right\rfloor} \mathcal{F}\left(q^{2^{k}}\right) .
$$

By the so-called children's binomial theorem ${ }^{\mathrm{c}}, \mathcal{F}(q) \mathcal{F}(q)=\mathcal{F}\left(q^{2}\right)$. Multiplying by $\mathcal{F}\left(q^{2}\right)$, we see that $\mathcal{F}(q) \mathcal{F}(q) \mathcal{F}\left(q^{2}\right)=\mathcal{F}\left(q^{2}\right) \mathcal{F}\left(q^{2}\right)=\mathcal{F}\left(q^{4}\right)$, and continuing we get

$$
\mathcal{F}(q) \mathcal{F}(q) \mathcal{F}\left(q^{2}\right) \mathcal{F}\left(q^{4}\right) \cdots \mathcal{F}\left(q^{2^{\left\lfloor\log _{2} n\right\rfloor}}\right)=\mathcal{F}\left(q^{2^{\left\lfloor\log _{2} n\right\rfloor+1}}\right) .
$$

Now notice that since $0<n<2^{\left\lfloor\log _{2} n\right\rfloor+1}$, the coefficient of $q^{n}$ on the right hand side of (4) is 0 , and our result follows.

We now give a list of recurrences for $\bar{f}_{n}$, discuss the usefulness of each, and prove them.

Lemma 2.2. If $\mathcal{F}(q) \overline{\mathcal{F}}(q)=1$, then $\bar{f}_{0}=1$ and for $n>0$,

i. $\bar{f}_{n}=\sum_{j=1}^{n} f_{j} \bar{f}_{n-j}$;

ii. $\bar{f}_{n}=1$ if and only if $\#\left\{\left(x_{0}, x_{1}, \ldots\right): x_{i} \in F, n=\sum_{i>0} x_{i} 2^{i}\right\}$ is odd;

iii. $\bar{f}_{n}=\sum_{\vec{x}} f_{x_{1}} f_{x_{2}} \cdots f_{x_{\ell}}$, where the summation extends over all tuples $\vec{x}=\left(x_{1}, \cdots, x_{\ell}\right)$ with $n=\sum_{i=1}^{\ell} x_{i}$ and each $x_{i}>0$ ( $\ell$ is allowed to vary);

${ }^{\mathrm{c}}(a+b)^{2}=a^{2}+b^{2}(\bmod 2)$ 
iv. $\bar{f}_{n}=\sum_{0 \leq i<n / 4} f_{n-2 i} \bar{f}_{i}+G\left(f_{1}, f_{2}, \ldots, f_{\lfloor n / 2\rfloor}\right)$, for some function $G$.

Lemma 2.2 $i$ i) is valuable because of its simplicity. For instance, it is immediately apparent from this recurrence relation that $\overline{\mathcal{F}}$ is uniquely defined and always exists (provided $f_{0}=1$ ).

In several of the examples we consider, the set $F$ has some special properties modulo a power of 2. Lemma 2.2 $(i i)$ facilitates our exploitation of these special properties.

Lemma 2.2 $(\mathrm{iii})$ is useful because of its symmetry, and because its right-hand side does not expressly reference the $\bar{f}$ sequence. As a specific example, let $r(n)$ be the number of ways to write $n$ as a sum of positive pentagonal numbers (counting order). Then, by Lemma 2.2 $(i i i), p(n) \equiv r(n)$ $(\bmod 2)$. We also use Lemma 2.2 (iii), for example, to prove Lemma 2.2 (iv).

If one lets the $f_{i}$ be independent random variables, then the expression in Lemma $2.2(\mathrm{iv})$ contains a summation of weakly dependent random variables, and a deterministic function of $f_{1}, \ldots, f_{n / 2}$. This allows us to say something explicit about the distribution of the resulting random variable $f_{n}$ (see Theorem 3.1).

Another remarkable aspect of Lemma 2.2 $(\mathrm{iv})$ is that $\bar{f}_{n}$ does not depend in any way on $f_{n-1}, f_{n-3}, \ldots, f_{n-c}$, where $c$ is the largest odd number strictly less than $n / 2$. For example,

$$
\bar{f}_{11}=f_{11}+f_{9} f_{1}+f_{7} f_{2}+f_{7} f_{1}+f_{5} f_{3}+f_{4} f_{3}+f_{3} f_{2} f_{1}+f_{3} f_{2}+f_{2} f_{1}+f_{1}
$$

does not depend on $f_{10}, f_{8}$, or $f_{6}$.

Proof. Comparing the coefficients of $q^{n}$ on the left- and right-hand sides of equation (2) yields $\sum_{j=0}^{n} f_{j} \bar{f}_{n-j}=0$. Lemma $2.2(i)$ is this expression rearranged, using the fact that $f_{0}=1$.

Similarly, Lemma 2.2 ii) equates the coefficients of $q^{n}$ on the left- and right-hand sides of equation (3), with the right-hand side interpreted as a product in $\mathbb{Z}$.

One can prove Lemma 2.2 (iii) by induction, using Lemma 2.2 $(i)$ to complete the induction step. Alternatively, one may simply compare the coefficients of $q^{n}$ on the left- and right-hand sides of

$$
\overline{\mathcal{F}}=\frac{1}{\mathcal{F}}=\frac{1}{1-(\mathcal{F}-1)}=1+(\mathcal{F}-1)+(\mathcal{F}-1)^{2}+(\mathcal{F}-1)^{3}+\cdots,
$$

which is valid because we are working over $\mathbb{F}_{2}$.

Recall Kummer's result that the multinomial coefficient $\left(\begin{array}{c}m_{1}+\cdots+m_{k} \\ m_{1}, m_{2}, \ldots, m_{k}\end{array}\right)=\frac{\left(m_{1}+\cdots+m_{k}\right) !}{m_{1} ! m_{2} ! \cdots m_{k} !}$ is relatively prime to a prime $p$ if and only if $m_{1}, \ldots, m_{k}$ can be added in base $p$ without carrying [10]. We are working with $p=2$, so our condition is: $\left(\begin{array}{c}m_{1}+\cdots+m_{k} \\ m_{1}, \ldots, m_{k}\end{array}\right)$ is odd if and only if no two of the binary expansions of $m_{1}, \ldots, m_{k}$ have a " 1 " in the same position. We call such a list of positive integers $m_{1}, \ldots, m_{k}$ non-overlapping.

Let $\pi(n)$ be the set of partitions of $n$ whose distinct parts $x_{1}, \ldots, x_{k}$ have non-overlapping 
multiplicities $m_{1}, \ldots, m_{k}$. Continuing from [2.2 $(i i i)$, we have

$$
\begin{aligned}
\bar{f}_{n} & =\sum_{\substack{x_{1}+\cdots+x_{\ell}=n \\
x_{i}>0}} f_{x_{1}} f_{x_{2}} \cdots f_{x_{\ell}} \\
& =\sum_{\substack{m_{1} a_{1}+\cdots+m_{k} a_{k}=n \\
a_{1}>\cdots>a_{k}>0 \\
m_{i}>0}}\left(\begin{array}{c}
m_{1}+\cdots+m_{k} \\
m_{1}, \ldots, m_{k}
\end{array}\right) f_{a_{1}}^{m_{1}} f_{a_{2}}^{m_{2}} \cdots f_{a_{k}}^{m_{k}} \\
& =\sum_{\substack{\pi(n) \\
a_{1}}} f_{a_{2}}^{m_{1}} f_{a_{2}}^{m_{2}} \cdots f_{a_{k}}^{m_{k}} \\
& =\sum_{\substack{\pi(n) \\
a_{1}>n / 2}} f_{a_{1}}^{m_{1}} f_{a_{2}}^{m_{2}} \cdots f_{a_{k}}^{m_{k}}+\sum_{\substack{\pi(n) \\
a_{1} \leq n / 2}} f_{a_{1}}^{m_{1}} f_{a_{2}}^{m_{2}} \cdots f_{a_{k}}^{m_{k}}
\end{aligned}
$$

If $a_{1}>n / 2$, then it must have multiplicity $m_{1}=1$, and if $m_{1}, \ldots, m_{k}$ are non-overlapping then the other $m_{i}$ are even:

$$
f_{a_{1}}^{m_{1}} f_{a_{2}}^{m_{2}} \cdots f_{a_{k}}^{m_{k}}=f_{a_{1}}\left(f_{a_{2}}^{m_{2} / 2} \cdots f_{a_{k}}^{m_{k} / 2}\right)^{2} .
$$

This implies that $n-a_{1}$ is even, and $a_{2} \frac{m_{2}}{2}+\cdots+a_{k} \frac{m_{k}}{2}$ is a partition of $\left(n-a_{1}\right) / 2$. Setting $2 i=n-a_{1}$, we get

$$
\bar{f}_{n}=\sum_{\pi(n)} f_{a_{1}}^{m_{1}} f_{a_{2}}^{m_{2}} \cdots f_{a_{k}}^{m_{k}}=\sum_{0 \leq i<n / 4} f_{n-2 i} \sum_{\pi(i)} f_{a_{1}}^{m_{1}} \cdots f_{a_{k}}^{m_{k}}+\sum_{\substack{\pi(n) \\ a_{i} \leq n / 2}} f_{a_{1}}^{m_{1}} \cdots f_{a_{k}}^{m_{k}} .
$$

Using Lemma 2.2 (iii) again, this becomes

$$
\bar{f}_{n}=\sum_{0 \leq i<n / 4} f_{n-2 i} \bar{f}_{i}+G\left(f_{1}, \ldots, f_{\lfloor n / 2\rfloor}\right)
$$

for a specific function $G$.

\section{$3 \quad$ Random power series}

In this section, we consider the reciprocal of a random power series in $\mathbb{F}_{2}[[q]]$. The results of this section are strong evidence that the density of $\mathcal{F}$ plays little to no role in determining the density of $\overline{\mathcal{F}}$, and that unless the coefficients of $\mathcal{F}$ have some structure, the density of $\overline{\mathcal{F}}$ is $1 / 2$.

Recall that a Bernoulli variable is a random variable that takes values in $\{0,1\}$.

Theorem 3.1. Suppose that $f_{1}, f_{2}, \ldots$ are independent Bernoulli variables, with

$$
\inf _{n} \min \left\{\mathbb{P}\left[f_{n}=0\right], \mathbb{P}\left[f_{n}=1\right]\right\}>0 .
$$

Then $\delta(\overline{\mathcal{F}})=1 / 2$ with probability 1 .

We need the following two lemmas. 
Lemma 3.2 (Lévy's Borel-Cantelli lemma). Let $E_{1}, E_{2}, \ldots$, be events, and define $Z_{n}:=$ $\sum_{k=1}^{n} I_{E_{k}}$, the random variable that records the number of $E_{1}, E_{2}, \ldots, E_{n}$ that occur. Define

$$
\xi_{k}:=\mathbb{P}\left[E_{k} \mid E_{1}, E_{2}, \ldots, E_{k-1}\right]
$$

If $\sum_{k=1}^{\infty} \xi_{k}$ diverges, then $Z_{n}$ is asymptotically equal to $\sum_{k=1}^{n} \xi_{k}$ with probability 1.

For a proof of Lévy's Borel-Cantelli lemma, we refer the reader to [14 Sec 12.15].

Lemma 3.3 (Binary Central Limit Theorem). Let $X_{i}$ be 0 with probability $\gamma_{i}$ and 1 with probability $1-\gamma_{i}$, and suppose that $X_{1}, X_{2}, \ldots$ are independent. Then, as $n \rightarrow \infty$,

$$
\mathbb{P}\left[\sum_{i=1}^{n} X_{i} \equiv 0 \quad(\bmod 2)\right] \rightarrow \frac{1}{2}
$$

if and only if some $\gamma_{i}=1 / 2$ or $\sum_{i=1}^{n} \min \left\{\gamma_{i}, 1-\gamma_{i}\right\}$ diverges.

Proof. Let $S_{n}:=\sum_{i=1}^{n} X_{i}$, and define $p_{i}$ by $\mathbb{P}\left[S_{n} \equiv 0(\bmod 2)\right]=p_{i}$. Clearly $S_{n}$ is even if and only if $S_{n-1}$ and $X_{n}$ are both even or both odd:

$$
p_{n}=p_{n-1} \gamma_{n}+\left(1-p_{n-1}\right)\left(1-\gamma_{n}\right) .
$$

Clearly $2 p_{1}-1=2 \gamma_{1}-1$, and

$$
2 p_{n}-1=2\left(p_{n-1} \gamma_{n}+\left(1-p_{n-1}\right)\left(1-\gamma_{n}\right)\right)-1=\left(2 p_{n-1}-1\right)\left(2 \gamma_{n}-1\right),
$$

which provides the base case and inductive step for the equality

$$
2 p_{n}-1=\prod_{i=1}^{n}\left(2 \gamma_{i}-1\right)
$$

By the standard results for infinite products, we now see that $2 p_{n}-1 \rightarrow 0$ if and only if $2 \gamma_{i}-1=0$ for some $i$ or $\sum_{i=1}^{n} \min \left\{\gamma_{i}, 1-\gamma_{i}\right\}$ diverges.

Proof of Theorem 3.1. We begin with some notation:

$$
\begin{aligned}
\alpha_{n} & :=\mathbb{P}\left[f_{n}=0\right], \\
\beta_{n} & :=\min \left\{\alpha_{n}, 1-\alpha_{n}\right\}, \\
\beta & :=\inf _{n \rightarrow \infty} \beta_{n},
\end{aligned}
$$

and note that $0<\beta \leq 1 / 2$. We will show first that $\mathbb{P}\left[\bar{f}_{n}=0\right] \rightarrow 1 / 2$ as $n \rightarrow \infty$, and then will show that $\delta(\bar{F})=1 / 2$ with probability 1 .

Lemma $\left[2.2(i)\right.$ says that $\bar{f}_{n}=f_{n}+\sum_{j=1}^{n-1} f_{j} \bar{f}_{n-j}$, whence

$$
\mathbb{P}\left[\bar{f}_{n}=0\right]=\mathbb{P}\left[f_{n}=0\right] \mathbb{P}\left[\sum_{j=1}^{n-1} f_{j} \bar{f}_{n-j}=0\right]+\mathbb{P}\left[f_{n}=1\right] \mathbb{P}\left[\sum_{j=1}^{n-1} f_{j} \bar{f}_{n-j}=1\right]
$$

is a weighted average of $\mathbb{P}\left[f_{n}=0\right]=\alpha_{n}$ and $\mathbb{P}\left[f_{n}=1\right]=1-\alpha_{n}$. Consequently, $\mathbb{P}\left[\bar{f}_{n}=0\right] \geq \beta_{n} \geq \beta$ and $\mathbb{P}\left[\bar{f}_{n}=1\right] \geq \beta_{n} \geq \beta$. 
Set $B_{n}:=\left\{i: 0 \leq i<n / 4, \bar{f}_{i}=1\right\}$, and set $G_{n}:=G\left(f_{1}, \ldots, f_{\lfloor n / 2\rfloor}\right)$, where $G$ is the function from Lemma 2.2 $i v)$. We have, from Lemma 2.2 $(i v)$,

$$
\bar{f}_{n}=\sum_{i \in B_{n}} f_{n-2 i}+G_{n}
$$

From the previous paragraph, we know that $\mathbb{E}\left[\left|B_{n}\right|\right]$ is at least $\sum_{i=0}^{\lfloor n / 4\rfloor} \beta_{i} \geq \beta\lfloor n / 4\rfloor$. In particular, a routine calculation shows that $\left|B_{n}\right| \rightarrow \infty$ with probability 1 . Thus, $\mathbb{P}\left[\left|B_{n}\right|>K_{n}\right] \rightarrow 1$ if $K_{n}$ goes to infinity sufficiently slowly. We have

$$
\mathbb{P}\left[\bar{f}_{n}=0\right]=\operatorname{P}\left[\bar{f}_{n}=0|| B_{n} \mid>K_{n}\right] \mathbb{P}\left[\left|B_{n}\right|>K_{n}\right]+\mathbb{P}\left[\bar{f}_{n}=0|| B_{n} \mid \leq K_{n}\right] \mathbb{P}\left[\left|B_{n}\right| \leq K_{n}\right],
$$

which for large $n$ becomes $\mathbb{P}\left[\bar{f}_{n}=0\right]=\mathbb{P}\left[\bar{f}_{n}=0|| B_{n} \mid>K_{n}\right]$.

We now observe that $\bar{f}_{n}=0$ if and only if $G_{n}=\sum_{i \in B_{n}} f_{n-2 i}$ (call this sum $\sigma_{n}$ ), so that

$$
\begin{aligned}
\mathbb{P}\left[\bar{f}_{n}=0|| B_{n} \mid>K_{n}\right] & = \\
\mathbb{P}\left[G_{n}=0|| B_{n} \mid>K_{n}, \sigma_{n}=0\right] & \mathbb{P}\left[\sigma_{n}=0|| B_{n} \mid>K_{n}\right]+ \\
& \mathbb{P}\left[G_{n}=1|| B_{n} \mid>K_{n}, \sigma_{n}=0\right] \mathbb{P}\left[\sigma_{n}=1|| B_{n} \mid>K_{n}\right] .
\end{aligned}
$$

This is a weighted average of $\mathbb{P}\left[\sigma_{n}=0|| B_{n} \mid>K_{n}\right]$ and $\mathbb{P}\left[\sigma_{n}=1|| B_{n} \mid>K_{n}\right]$, both of which go to $1 / 2$ as $n \rightarrow \infty$ by the Binary Central Limit Theorem. Thus,

$$
\mathbb{P}\left[\bar{f}_{n}=0\right] \approx \mathbb{P}\left[\bar{f}_{n}=0|| B_{n} \mid>K_{n}\right] \approx \frac{1}{2}
$$

with each of the " $\approx$ " becoming " $=$ " as $n \rightarrow \infty$.

Now that we have shown that $\mathbb{P}\left[\bar{f}_{n}=0\right] \rightarrow 1 / 2$, we know that $\mathbb{E}[\delta(\bar{F}, n)] \rightarrow 1 / 2$, but this does not imply that $\delta(\bar{F}, n) \rightarrow 1 / 2$ ever, much less with probability 1 . This last step again requires the at-least-weak independence of $\bar{f}_{n}$ from $\bar{f}_{1}, \ldots, \bar{f}_{n-1}$, and the technicalities are handled for us by Lévy's Borel-Cantelli lemma.

Let $E_{k}$ be the event $\left\{\bar{f}_{k}=0\right\}$, and set $\xi_{k}:=\mathbb{P}\left[\bar{f}_{k}=0 \mid \bar{f}_{1}, \bar{f}_{2}, \ldots, \bar{f}_{n}\right]$. By the comment above, $0<\beta \leq \xi_{k}$, so $\sum_{k=1}^{\infty} \xi_{k}=\infty$. Thus, by Lemma 3.2

$$
\lim _{n \rightarrow \infty} \frac{\delta(\bar{F}, n)}{\frac{1}{n} \sum_{k=1}^{n} \xi_{k}}=1
$$

with probability 1. For every $\epsilon>0$ there is an $n_{0}$ such that for all $n>n_{0}$

$$
(1-\epsilon) \frac{1}{n} \sum_{k=1}^{n} \xi_{k} \leq \delta(\bar{F}, n) \leq(1+\epsilon) \frac{1}{n} \sum_{k=1}^{n} \xi_{k} .
$$

These upper and lower bounds on $\delta(\bar{F}, n)$ are non-random, so we may take expectations (for large $n$ ) to get

$$
(1-\epsilon) \frac{1}{n} \sum_{k=1}^{n} \xi_{k} \leq \frac{1}{2} \leq(1+\epsilon) \frac{1}{n} \sum_{k=1}^{n} \xi_{k},
$$


where we have used the linearity of expectation and the previously proved $\mathbb{E}\left[\bar{f}_{n}=0\right]=\mathbb{P}\left[\bar{f}_{n}=0\right] \rightarrow$ $1 / 2$. This implies that $\frac{1}{n} \sum_{k=1}^{n} \xi_{k} \rightarrow 1 / 2$ also. Consequently, (5) now implies that

$$
\delta(\bar{F}):=\lim _{n \rightarrow \infty} \delta(\bar{F}, n)=\frac{1}{2}
$$

with probability 1.

\section{Polynomials}

In this section, we study the reciprocals of polynomials in $\mathbb{F}_{2}[q]$. The coefficients of such a reciprocal are periodic (see Proposition 4.3 below), and so the reciprocal has rational density. We also give some indication of how the densities of reciprocals of polynomials are distributed, beginning in Subsection 4.1 In Subsection 4.2 we use the theory of de Bruijn cycles to exhibit an infinite family of polynomials whose reciprocals have densities strictly larger than $1 / 2$; in Subsection 4.3 we show that if two polynomials have product $1+q^{D}(D \geq 4)$, then at least one of them has a reciprocal with density at most $1 / 2$. In Subsection 4.4 we show that the reciprocal of an eventually periodic set $^{\mathrm{d}}$ containing 0 is an eventually periodic set containing 0 .

Let $\sum_{i=0}^{\infty} b_{i} 2^{i}$ be the binary expansion of $n$; we define the polynomial $\mathcal{P}_{n}(q):=\sum_{i=0}^{\infty} b_{i} q^{i} \in \mathbb{F}_{2}[q]$. Clearly this indexes all polynomials, and the invertible polynomials are precisely those with $n$ odd. For a polynomial $\mathcal{Q} \in \mathbb{F}_{2}[q]$, we let $\hat{\mathcal{Q}}$ be the same polynomial with coefficients (all 0 or 1 ) in $\mathbb{Z}$. For instance, $\mathcal{Q}=\mathcal{P}_{\hat{\mathcal{Q}}(2)}$ for every polynomial $\mathcal{Q}$.

We denote by $\ell(\mathcal{P})$ the length of the polynomial, i.e., $\ell(\mathcal{P})=\hat{\mathcal{P}}(1)$, and by $\operatorname{deg}(\mathcal{P})$ the degree of the polynomial. Also, $\operatorname{ord}(\mathcal{P})$ is the least positive $D$ such that $\mathcal{P}$ divides $1+q^{D}$. It is not immediately obvious that $\operatorname{ord}(\mathcal{P})$ is well defined: it is for invertible $\mathcal{P}$, and this is the content of Proposition 4.1 below. For each polynomial $\mathcal{P}$, we define $\mathcal{P}^{*}$ by $\mathcal{P} \mathcal{P}^{*}=1+q^{\text {ord } \mathcal{P}}$. We shall see that the properties of $\mathcal{P}$ and $\mathcal{P}^{*}$ are intimately related (Propositions 4.2 and 4.5).

If $\operatorname{ord}(\mathcal{P})=2^{\operatorname{deg}(\mathcal{P})}-1$, then $\mathcal{P}$ is called primitive, and $\mathbb{F}_{2}[q] /(\mathcal{F})$ is isomorphic to $\mathbb{F}_{2 \operatorname{deg}(\mathcal{P})}$, with multiplicative generator $q$. All primitive polynomials are irreducible, but not vice versa; for example $1+q^{3}+q^{6}$ and $1+q+q^{2}+q^{3}+q^{4}$ are irreducible but not primitive.

Figure 3 tabulates properties of $\mathcal{P}_{n}$ for odd $n<256$, including factorizations, $\mathcal{P}_{n}^{*}$, and densities of reciprocals.

In Figure 4 we plot the points $\left(n, \delta\left(\overline{\mathcal{P}}_{n}\right)\right)$ for odd $n$ less than $2^{12}$. We note that $\delta\left(\overline{\mathcal{P}}_{n}\right)$ tends to be near $1 / 2$, but is biased toward being below $1 / 2$. This is also suggested, but not proven, by Proposition 4.5 below. In Proposition 4.4 we give an algebraically-described infinite set of $n$ such that $\delta\left(\overline{\mathcal{P}}_{n}\right)>1 / 2$. Note that $\delta\left(\overline{\mathcal{P}}_{n}\left(q^{k}\right)\right)=\frac{1}{k} \delta\left(\overline{\mathcal{P}}_{n}(q)\right)$, i.e., if $\mathcal{P}_{n}$ is a polynomial in $q^{2}, q^{3}$, etc, then its density is a priori less than $1 / 2,1 / 3$, etc. These points have been plotted with squares.

In Figure 5 we plot the empirical distribution function of $\delta\left(\overline{\mathcal{P}}_{n}\right)$. The large discontinuities near $1 / 2$ mean that these densities occur with large frequency (fully 421 of the 2048 polynomials $\mathcal{P}_{1}, \mathcal{P}_{3}, \ldots, \mathcal{P}_{4095}$ have reciprocals with density exactly $\left.1 / 2\right)$. Again visible in Figure 5 is the preference of $\mathcal{P}$ to have reciprocal with density less than $1 / 2$. The most interesting issue raised in this section, which remains unanswered, is to describe the set

$$
\{\delta(\overline{\mathcal{P}}): \mathcal{P} \text { is a polynomial }\} .
$$

For example, is there an $n$ with $\delta\left(\overline{\mathcal{P}}_{n}\right)=3 / 4$ ?

\footnotetext{
${ }^{\mathrm{d}}$ More precisely, a set whose indicator function is eventually periodic.
} 


\begin{tabular}{|c|c|c|c|c|c|c|c|c|}
\hline$n$ & $\hat{\mathcal{P}}^{*}(2)$ & $D$ & Factors & $\delta\left(\overline{\mathcal{P}}_{n}\right)$ & $n$ & $D$ & Factors & $\delta\left(\overline{\mathcal{P}}_{n}\right)$ \\
\hline 1 & 1 & 1 & prim & 0 & 129 & $\begin{array}{lll}7 & \end{array}$ & $3 \cdot 11 \cdot 13$ & $1 / 7$ \\
\hline 3 & 1 & 1 & prim & 1 & 131 & 127 & prim & $64 / 127$ \\
\hline 5 & 1 & 2 & $3^{2}$ & $1 / 2$ & 133 & 93 & $7 \cdot 55$ & $46 / 93$ \\
\hline 7 & 3 & 3 & prim & $2 / 3$ & 135 & 60 & $3^{3} \cdot 25$ & $1 / 2$ \\
\hline 9 & 1 & 3 & $3 \cdot 7$ & $1 / 3$ & 137 & 127 & prim & $64 / 127$ \\
\hline 11 & 23 & 7 & prim & $4 / 7$ & 139 & 15 & $3 \cdot 7 \cdot 19$ & $1 / 3$ \\
\hline 13 & 29 & 7 & prim & $4 / 7$ & 141 & 62 & $3^{2} \cdot 41$ & $1 / 2$ \\
\hline 15 & 3 & 4 & $3^{3}$ & $1 / 2$ & 143 & 127 & prim & $64 / 127$ \\
\hline 17 & 1 & 4 & $3^{4}$ & $1 / 4$ & 145 & 127 & prim & $64 / 127$ \\
\hline 19 & 2479 & 15 & prim & $8 / 15$ & 147 & 62 & $3^{2} \cdot 47$ & $1 / 2$ \\
\hline 21 & 5 & 6 & $7^{2}$ & $1 / 3$ & 149 & 63 & $3 \cdot 115$ & $31 / 63$ \\
\hline 23 & 11 & 7 & $3 \cdot 13$ & $3 / 7$ & 151 & 42 & $7^{2} \cdot 11$ & $10 / 21$ \\
\hline 25 & 3929 & 15 & prim & $8 / 15$ & 153 & 24 & $3^{5} \cdot 7$ & $1 / 2$ \\
\hline 27 & 7 & 6 & $3^{2} \cdot 7$ & $1 / 2$ & 155 & 35 & prim & $18 / 35$ \\
\hline 29 & 13 & 7 & $3 \cdot 11$ & $3 / 7$ & 157 & 127 & prim & $64 / 127$ \\
\hline 31 & 3 & 5 & irr & $2 / 5$ & 159 & 21 & $3 \cdot 117$ & $3 / 7$ \\
\hline 33 & 1 & 5 & $3 \cdot 31$ & $1 / 5$ & 161 & 93 & $7 \cdot 59$ & $46 / 93$ \\
\hline 35 & 72031 & 21 & $7 \cdot 13$ & $10 / 21$ & 163 & 63 & $3 \cdot 97$ & $31 / 63$ \\
\hline 37 & 78898037 & 31 & prim & $16 / 31$ & 165 & 20 & $3^{3} \cdot 31$ & $1 / 2$ \\
\hline 39 & 635 & 14 & $3^{2} \cdot 11$ & $1 / 2$ & 167 & 127 & prim & $64 / 127$ \\
\hline 41 & 91635305 & 31 & prim & $16 / 31$ & 169 & 63 & $3 \cdot 103$ & $31 / 63$ \\
\hline 43 & 1335 & 15 & $3 \cdot 25$ & $7 / 15$ & 171 & 127 & prim & $64 / 127$ \\
\hline 45 & 189 & 12 & $3^{3} \cdot 7$ & $1 / 2$ & 173 & 105 & $11 \cdot 19$ & $52 / 105$ \\
\hline 47 & 94957459 & 31 & prim & $16 / 31$ & 175 & 42 & $3^{2} \cdot 7 \cdot 13$ & $1 / 2$ \\
\hline 49 & 128305 & 21 & $7 \cdot 11$ & $10 / 21$ & 177 & 62 & $3^{2} \cdot 37$ & $1 / 2$ \\
\hline 51 & 15 & 8 & $3^{5}$ & $1 / 2$ & 179 & 93 & $7 \cdot 61$ & $46 / 93$ \\
\hline 53 & 1893 & 15 & $3 \cdot 19$ & $7 / 15$ & 181 & 105 & $13 \cdot 25$ & $52 / 105$ \\
\hline 55 & 121098539 & 31 & prim & $16 / 31$ & 183 & 63 & $3 \cdot 109$ & $31 / 63$ \\
\hline 57 & 889 & 14 & $3^{2} \cdot 13$ & $1 / 2$ & 185 & 127 & prim & $64 / 127$ \\
\hline 59 & 111435623 & 31 & prim & $16 / 31$ & 187 & 28 & $3^{4} \cdot 11$ & $13 / 28$ \\
\hline 61 & 105887917 & 31 & prim & $16 / 31$ & 189 & 12 & $3 \cdot 7^{3}$ & $1 / 3$ \\
\hline 63 & 3 & 6 & $3 \cdot 7^{2}$ & $1 / 3$ & 191 & 127 & prim & $64 / 127$ \\
\hline 65 & 1 & 6 & $3^{2} \cdot 7^{2}$ & $1 / 6$ & 193 & 127 & prim & $64 / 127$ \\
\hline 67 & 151054908502416063 & 63 & prim & $32 / 63$ & 195 & 12 & $3^{3} \cdot 7^{2}$ & $1 / 2$ \\
\hline 69 & 277 & 14 & $11^{2}$ & $2 / 7$ & 197 & 63 & $3 \cdot 67$ & $31 / 63$ \\
\hline 71 & 37394331 & 31 & $3 \cdot 61$ & $15 / 31$ & 199 & 105 & $13 \cdot 19$ & $52 / 105$ \\
\hline 73 & 9 & 9 & irr & $2 / 9$ & 201 & 62 & $3^{2} \cdot 61$ & $1 / 2$ \\
\hline 75 & 4865751 & 28 & $3^{3} \cdot 13$ & $1 / 2$ & 203 & 127 & prim & $64 / 127$ \\
\hline 77 & 40094429 & 31 & $3 \cdot 59$ & $15 / 31$ & 205 & 93 & $7 \cdot 47$ & $46 / 93$ \\
\hline 79 & 627 & 15 & $7 \cdot 25$ & $2 / 5$ & 207 & 14 & $3 \cdot 11^{2}$ & $3 / 7$ \\
\hline 81 & 337 & 14 & $13^{2}$ & $2 / 7$ & 209 & 15 & $3 \cdot 7 \cdot 25$ & $1 / 3$ \\
\hline 83 & 44271 & 21 & prim & $11 / 21$ & 211 & 127 & prim & $64 / 127$ \\
\hline 85 & 5 & 8 & $3^{6}$ & $1 / 4$ & 213 & 127 & prim & $64 / 127$ \\
\hline 87 & 42187 & 21 & irr & $8 / 21$ & 215 & 62 & $3^{2} \cdot 59$ & $1 / 2$ \\
\hline 89 & 49106713 & 31 & $3 \cdot 55$ & $15 / 31$ & 217 & 35 & prim & $18 / 35$ \\
\hline 91 & 215232491192501383 & 63 & prim & $32 / 63$ & 219 & 9 & $3 \cdot 73$ & $1 / 3$ \\
\hline 93 & 717 & 15 & $7 \cdot 31$ & $2 / 5$ & 221 & 28 & $3^{4} \cdot 13$ & $13 / 28$ \\
\hline 95 & 24018211 & 30 & $3^{2} \cdot 19$ & $1 / 2$ & 223 & 93 & $7 \cdot 41$ & $46 / 93$ \\
\hline 97 & 285247320157033569 & 63 & prim & $32 / 63$ & 225 & 60 & $3^{3} \cdot 19$ & $1 / 2$ \\
\hline 99 & 31 & 10 & $3^{2} \cdot 31$ & $1 / 2$ & 227 & 105 & $11 \cdot 25$ & $52 / 105$ \\
\hline 101 & 63285 & 21 & prim & $11 / 21$ & 229 & 127 & prim & $64 / 127$ \\
\hline 103 & 272840796136989499 & 63 & prim & $32 / 63$ & 231 & 15 & $3 \cdot 7 \cdot 31$ & $7 / 15$ \\
\hline 105 & 7716393 & 28 & $3^{3} \cdot 11$ & $1 / 2$ & 233 & 42 & $7^{2} \cdot 13$ & $10 / 21$ \\
\hline 107 & 119 & 12 & $7^{3}$ & $1 / 2$ & 235 & 62 & $3^{2} \cdot 55$ & $1 / 2$ \\
\hline 109 & 253483157574931709 & 63 & prim & $32 / 63$ & 237 & 63 & $3 \cdot 91$ & $31 / 63$ \\
\hline 111 & 59858643 & 31 & $3 \cdot 37$ & $15 / 31$ & 239 & 127 & prim & $64 / 127$ \\
\hline 113 & 57124209 & 31 & $3 \cdot 47$ & $15 / 31$ & 241 & 127 & prim & $64 / 127$ \\
\hline 115 & 248574834945763919 & 63 & prim & $32 / 63$ & 243 & 14 & $3 \cdot 13^{2}$ & $3 / 7$ \\
\hline 117 & 54053 & 21 & irr & $8 / 21$ & 245 & 42 & $3^{2} \cdot 7 \cdot 11$ & $1 / 2$ \\
\hline 119 & 107 & 12 & $3^{4} \cdot 7$ & $5 / 12$ & 247 & 127 & & \\
\hline 121 & 825 & 15 & $7 \cdot 19$ & $2 / 5$ & 249 & 21 & $3 \cdot 87$ & $3 / 7$ \\
\hline 123 & 53340711 & 31 & $3 \cdot 41$ & $15 / 31$ & 251 & 93 & $7 \cdot 37$ & $46 / 93$ \\
\hline 125 & 25787629 & 30 & $3^{2} \cdot 25$ & $1 / 2$ & 253 & 127 & prim & $64 / 127$ \\
\hline 127 & 3 & 7 & $11 \cdot 13$ & $2 / 7$ & 255 & 8 & $3^{7}$ & $1 / 4$ \\
\hline
\end{tabular}

Figure 3: Properties of $\mathcal{P}_{n}$ for odd $n<256$. The "Factors" column records whether $\mathcal{P}_{n}$ is reducible, irreducible or primitive. If $\mathcal{P}_{n}$ is reducible, then the factors column evaluates the factors of $\mathcal{P}_{n}$ at $q=2$. For example, the factors of $P_{245}$ are given as $3^{2} \cdot 7 \cdot 11$, whence $P_{245}=P_{3}^{2} P_{7} P_{11}$. 


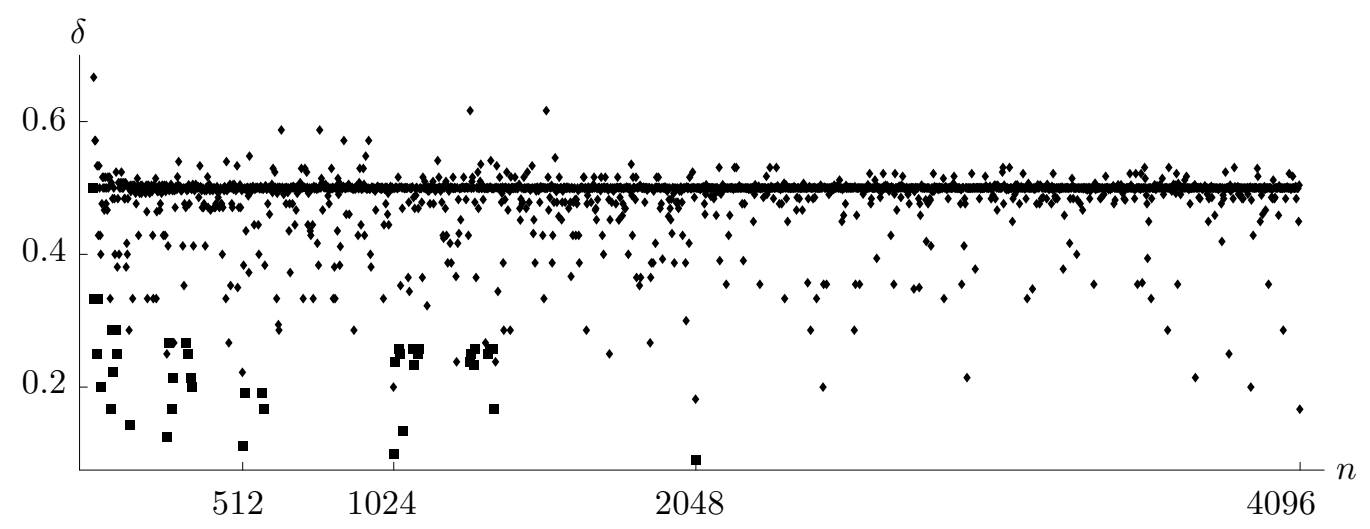

Figure 4: The points $\left(n, \delta\left(\overline{\mathcal{P}}_{n}\right)\right)$ with $n$ odd, except $(1,0)$ and $(3,1)$

\subsection{Order and Density}

Our first proposition demonstrates that $\operatorname{ord}(\mathcal{P})$ is well-defined, and our next proposition shows the connection between $\delta(\overline{\mathcal{P}})$, ord $(\mathcal{P})$, and $\mathcal{P}^{*}$.

Proposition 4.1. If $\mathcal{P}$ is a polynomial, then $\operatorname{ord}(\mathcal{P})$ is finite. ${ }^{\mathrm{e}}$

Proof. Let $\mathcal{V}_{1}, \ldots, \mathcal{V}_{k}$ be the irreducible factors of $\mathcal{P}$, and let $d_{i}$ be the multiplicative order of $q$ in the field $\mathbb{F}_{2}[q] /\left(\mathcal{V}_{i}\right)$. In particular, $1+q^{x d_{i}}$ is a multiple of $\mathcal{V}_{i}$ for each $x \in \mathbb{N}$. Set $L:=\operatorname{lcm}\left\{d_{1}, \ldots, d_{k}\right\}$ and define $\mathcal{V}_{i}^{*}$ by $\mathcal{V}_{1} \mathcal{V}_{1}^{*}=1+q^{L}$ and for $1<i \leq k$ by $\mathcal{V}_{i} \mathcal{V}_{i}^{*}=1+q^{2^{i-2} L}$. Now

$$
\begin{aligned}
\mathcal{P} \cdot \prod_{i=1}^{k} \mathcal{V}_{i}^{*}=\left(1+q^{L}\right)\left(1+q^{L}\right) & \left(1+q^{2 L}\right) \cdots\left(1+q^{2^{k-2} L}\right) \\
= & \left(1+q^{2 L}\right)\left(1+q^{2 L}\right)\left(1+q^{4 L}\right) \cdots\left(1+q^{2^{k-2} L}\right)=\left(1+q^{2^{k-1} L}\right),
\end{aligned}
$$

by repeated use of the children's binomial theorem.

We emphasize that, given $\mathcal{P}$ and the equality $\mathcal{P} \mathcal{F}=1+q^{D}$ for some $\mathcal{F}, D$ is not uniquely determined. For example, $\mathcal{P}(q) \mathcal{P}(q) \mathcal{F}\left(q^{2}\right)=1+q^{2 D}$. Nor does the proof given above always provide the minimal $D$.

Proposition 4.2. $\delta(\overline{\mathcal{P}})=\ell\left(\mathcal{P}^{*}\right) / \operatorname{ord}(\mathcal{P})$.

Proof. Since $\mathcal{P} \frac{\mathcal{P}^{*}}{1+q^{\text {ord } \mathcal{P}}}=1$, we see that the reciprocal of $\mathcal{P}$ is periodic with period ord $\mathcal{P}$ (although this may not be the minimal period), and in each period has density $\ell\left(\mathcal{P}^{*}\right) / \operatorname{ord} \mathcal{P}$.

\section{2 de Bruijn cycle algebra}

Our next proposition shows that the reciprocal of a polynomial is a special case of a linear-shift register. Fortunately, there is an enormous literature on linear-shift registers (see [9], for example).

${ }^{\mathrm{e}}$ Actually, the proof can be refined to show that $\operatorname{ord}(\mathcal{P}) \mid 2^{\operatorname{deg}(\mathcal{P})}-1$ if $\mathcal{P}$ is irreducible, and otherwise $\operatorname{ord}(\mathcal{P})=$ $2^{i} \operatorname{lcm}\left\{\operatorname{ord}\left(\mathcal{V}_{1}\right), \ldots, \operatorname{ord}\left(\mathcal{V}_{k}\right)\right\}$ for some $1 \leq 2^{i} \leq k$, where $\mathcal{P}=\mathcal{V}_{1} \cdots \mathcal{V}_{k}$. 


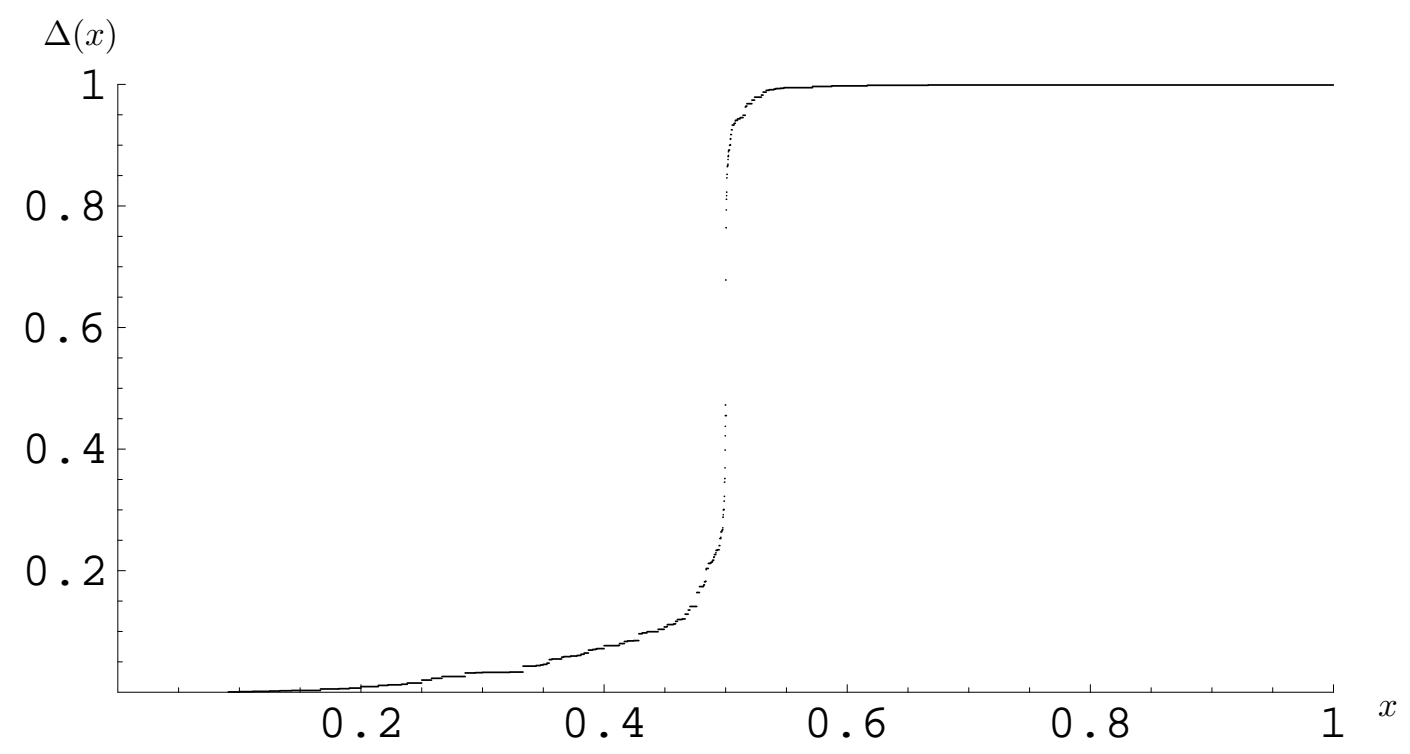

Figure 5: The distribution function $\Delta(x):=2^{-11} \cdot \#\left\{n: 1 \leq n \leq 2^{12}, n\right.$ odd, $\left.\delta\left(\overline{\mathcal{P}}_{n}\right) \leq x\right\}$

Proposition 4.3. If $\mathcal{F}$ is a polynomial with degree $d$, then (letting $\bar{f}_{j}=0$ for negative $j$ )

$$
\bar{f}_{n}=\sum_{j=1}^{d} f_{j} \bar{f}_{n-j} .
$$

Alternatively, $\bar{f}_{n}$ is the constant term of $q^{-n} \bmod \mathcal{F}$.

Proof. Since $f_{j}=0$ for all $j>d$, the recurrence (6) follows immediately from Lemma [2.2 $(i)$.

Let $M$ be the matrix whose $k^{\text {th }}$ row is the elementary vector supported in coordinate $k+1$, for $k=1, \ldots, d-1$, and whose last row is the vector $\left(f_{0}, \ldots, f_{d-1}\right)$, i.e., $M$ is the companion matrix of $\mathcal{F}$. Write $c_{n}$ for the constant coefficient of $q^{n} \bmod \mathcal{F}$. We claim that

$$
M\left(\begin{array}{c}
c_{s} \\
\vdots \\
c_{s+d-1}
\end{array}\right)=\left(\begin{array}{c}
c_{s+1} \\
\vdots \\
c_{s+d}
\end{array}\right) .
$$

To see this, let $Y_{k}$ denote scalar projection of elements of $\mathbb{F}_{2}[q] /(\mathcal{F})$ onto $q^{k}$, and let $X$ denote multiplication by $q$ in $\mathbb{F}_{2}[q] /(\mathcal{F})$. Both of these maps are linear, and it is easy to see that $Y_{k}=$ 


$$
\begin{aligned}
Y_{k-1} X^{-1} & +f_{k} Y_{0}, \text { for } 1 \leq k \leq d-1 . \text { Therefore } \\
Y_{0}= & Y_{d-1} X^{-1} \\
= & Y_{d-2} X^{-2}+f_{d-1} Y_{0} X^{-1} \\
& =Y_{d-3} X^{-3}+f_{d-2} Y_{0} X^{-2}+f_{d-1} Y_{0} X^{-1} \\
& \vdots \\
& =\sum_{j=0}^{d-1} f_{j} Y_{0} X^{d-j} .
\end{aligned}
$$

Applying $Y_{0}$ to $q^{s+d}$ yields $c_{s+d}=\sum_{j=0}^{d-1} f_{j} c_{s+j}$, which implies (7). Set $a_{n}:=c_{-n}$ (define $c$ on negative subscripts by using the recurrence). Thus, the sequences $\left(a_{n}\right)$ and $\left(\bar{f}_{n}\right)$ satisfy the same recurrence, with initial conditions $a_{0}=\bar{f}_{0}=1, c_{-i}=a_{i}=\bar{f}_{i}=0($ for $-d<i<0)$.

Our next proposition computes the density of the reciprocal of every primitive polynomial, and thereby produces an infinite family of polynomials whose reciprocals have density greater than $1 / 2$.

Proposition 4.4. If $\mathcal{P}$ is a primitive polynomial with degree $d$, then $\delta(\overline{\mathcal{P}})=\frac{2^{d-1}}{2^{d}-1}$.

Proof. A de Bruijn cycle of order $d$ is a binary sequence $\{S(n)\}_{n=1}^{q^{d}}$ in which every binary $d$-word appears in a "window" $(S(n+1), \ldots, S(n+d))$ for some $j$ (indices taken modulo $q^{d}$ ). A reduced de Bruijn cycle is a string of length $q^{d}-1$ which achieves every $d$-word in some window, except for the word $0^{d}$. Note that a reduced de Bruijn cycle may always be turned into an ordinary de Bruijn cycle by inserting an extra "0" into its longest run of 0's.

If $\mathcal{P}$ is primitive, then $q$ is a generator of $\mathbb{F}_{2^{d}}^{\times}$, and it is a classical result that the sequence of constant coefficients of the powers of a multiplicative generator yield a reduced de Bruijn cycle. Thus, by Proposition 4.3 the first $2^{d}-1$ coefficients of $\overline{\mathcal{P}}$ are a reduced binary de Bruijn cycle of order $d$. The reader wishing to explore de Bruijn cycles further can find the basics in 8,9 .

Since every string except $0^{d}$ appears in $\overline{\mathcal{P}}$, there are exactly $2^{d-1}$ ones in any period.

\subsection{Polynomials with non-high density reciprocals}

We see in Figure 4 that polynomials typically have reciprocals with density near $1 / 2$. In Figure 6 it is apparent that there is a connection between the density of $\overline{\mathcal{P}}$ and $\overline{\mathcal{P}}^{*}$. Our next theorem elucidates the connection.

Proposition 4.5. If $\operatorname{ord}(\mathcal{P}) \geq 4$, then $\min \left\{\delta(\overline{\mathcal{P}}), \delta\left(\overline{\mathcal{P}}^{*}\right)\right\} \leq 1 / 2$.

This proposition is best possible in that $\mathcal{P}_{51} \mathcal{P}_{15}=1+q^{8}$, and $\delta\left(\overline{\mathcal{P}}_{51}\right)=\delta\left(\overline{\mathcal{P}}_{15}\right)=1 / 2$.

Proof. Set $D:=\operatorname{ord}(\mathcal{P})$. We assume without loss of generality that $\operatorname{deg}(\mathcal{P}) \leq D / 2 \leq \operatorname{deg}\left(\mathcal{P}^{*}\right)$. If 


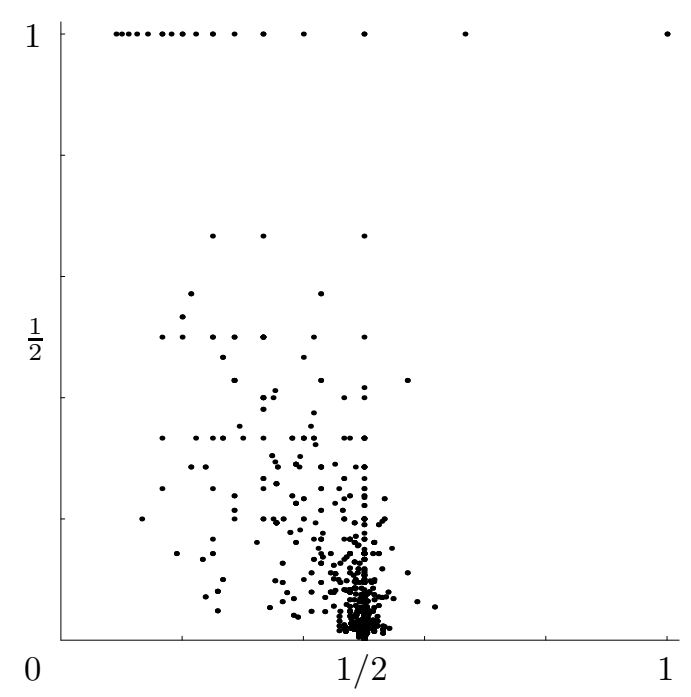

Figure 6: The points $\left(\delta\left(\overline{\mathcal{P}}_{n}\right), \delta\left(\overline{\mathcal{P}}_{n}^{*}\right)\right)$ for odd $n<2^{12}$

$\operatorname{deg}(\mathcal{P})<3$, then we appeal to the following table of calculations:

\begin{tabular}{|c|c|c|c|}
\hline $\mathcal{P}$ & $\mathcal{P}^{*}$ & $\overline{\mathcal{P}}^{*}$ & $\delta\left(\overline{\mathcal{P}}^{*}\right)$ \\
\hline \hline 1 & $1+q^{D}$ & $\sum_{n=0}^{\infty} q^{n D}$ & $1 / D$ \\
$1+q$ & $\sum_{n=0}^{D-1} q^{n}$ & $\sum_{n=0}^{\infty}\left(q^{n D}+q^{n D+1}\right)$ & $2 / D$ \\
$1+q^{2}$ & $\sum_{n=0}^{D-1} q^{2 n}$ & $\sum_{n=0}^{\infty}\left(q^{2 n D}+q^{2 n D+2}\right)$ & $1 / D$ \\
$1+q+q^{2}$ & $(1+q) \sum_{n=0}^{D / 3-1} q^{3 n}$ & $\sum_{n=0}^{\infty}\left(q^{n D}+q^{n D+1}+q^{n D+2}\right)$ & $3 / D$ \\
\hline
\end{tabular}

In the case $\mathcal{P}=1+q+q^{2}=\frac{1+q^{3}}{1+q}$, we see also that $D \equiv 0(\bmod 3)$, and by hypothesis $D \geq 4$, so that $D / 3 \leq 1 / 2$.

We assume now that $\operatorname{deg}(\mathcal{P}) \geq 3$. Since

$$
\overline{\mathcal{P}}^{*}=\frac{\mathcal{P}}{1+q^{D}}=\mathcal{P}+q^{D} \mathcal{P}+q^{2 D} \mathcal{P}+q^{3 D} \mathcal{P}+\cdots
$$

and $\operatorname{deg}(\mathcal{P})<D$, we have $\delta\left(\overline{\mathcal{P}}^{*}\right)=\ell(\mathcal{P}) / D$. If $\mathcal{P}$ has any zero coefficients, then $\ell(\mathcal{P}) \leq \operatorname{deg}(\mathcal{P}) \leq D / 2$ and so $\delta\left(\overline{\mathcal{P}}^{*}\right) \leq 1 / 2$. If $\mathcal{P}$ has no zero coefficients, then $\mathcal{P}=\left(1+q^{\operatorname{deg}(\mathcal{P})+1}\right) /(1+q)$, in which case $\overline{\mathcal{P}}=(1+q) /\left(1+q^{\operatorname{deg}(\mathcal{P})+1}\right)$, a series which has density $2 /(\operatorname{deg}(\mathcal{P})+1)$. Since $\operatorname{deg}(\mathcal{P}) \geq 3$, this quantity is $\leq 1 / 2$.

Corollary 4.6. If $\mathcal{P} \notin\left\{1,1+q, 1+q+q^{2}\right\}$ is a polynomial and $\mathcal{P}^{*}$ is primitive, then $\delta(\overline{\mathcal{P}}) \leq 1 / 2$.

\subsection{Eventually periodic sets}

An eventually periodic set is one whose generating function has the form $\mathcal{E}(q)+\frac{\mathcal{P}(q)}{1+q^{D}}$, for some polynomials $\mathcal{E}, \mathcal{P}$ with $\operatorname{deg}(\mathcal{P})<D$, and exactly one of $\mathcal{E}, \mathcal{P}$ has constant term 1 . The finite sets 
containing 0 are examples. Another example is given by the set $\mathbb{N} \backslash\{n: n \equiv 2(\bmod 4)\}$ (which has density $3 / 4$ ), whose reciprocal is the set $\{1\} \cup\{n \in \mathbb{N}: n$ is congruent to $0,2,5$, or 6 modulo 7$\}$ (which has density $4 / 7$ ).

Proposition 4.7. The reciprocal of an eventually periodic set is an eventually periodic set.

This proposition is essentially the same as that which asserts that rational numbers have eventually periodic decimal expansions.

Proof. Obviously, the reciprocal of a ratio of polynomials (each with constant term 1 ) is a ratio of polynomials (each with constant term 1). All that we need to observe is that such a ratio $\mathcal{R} / \mathcal{S}$ can be written in the form

$$
\frac{\mathcal{R}}{\mathcal{S}}=\mathcal{E}+\frac{\mathcal{Q}}{1+q^{D}}
$$

with $\operatorname{deg}(\mathcal{Q})<D$. By long division, we can write $\mathcal{R} / \mathcal{S}$ in the form $\mathcal{E}+\mathcal{P} / \mathcal{S}$ with $\operatorname{deg}(\mathcal{P})<\operatorname{deg}(\mathcal{S})$. But this is the same as $\mathcal{E}+\frac{\mathcal{P} \mathcal{S}^{*}}{1+q^{D}}$, where $\mathcal{S S}^{*}=1+q^{D}$, and $\operatorname{deg}\left(\mathcal{P} \mathcal{S}^{*}\right)<\operatorname{deg}\left(\mathcal{S} \mathcal{S}^{*}\right)=D$.

\section{The powers of two}

We saw in Section 4 that the reciprocal of a polynomial (other than $\mathcal{P}_{1}$ ) has positive density. One might wonder if the reciprocal of any set with zero density has positive density. Our next theorem shows that this is not the case.

We note the $m=1$ case of Theorem [5.1] the reciprocal of $A_{1}=\{0\} \cup\left\{2^{n}: n \in \mathbb{N}\right\}$ is $\bar{A}_{1}=$ $\left\{2^{n}-1: n \in \mathbb{N}\right\}$. This is easily proved directly by considering the following sum-preserving involution on $A_{1} \times \bar{A}_{1}$. For $s, t \in \mathbb{N}$ and distinct, define $\mu(0,0)=(0,0), \mu\left(0,2^{t+1}-1\right)=\left(2^{t}, 2^{t}-1\right), \mu\left(2^{s}, 2^{t}-1\right)=$ $\left(2^{t}, 2^{s}-1\right), \mu\left(2^{t}, 2^{t}-1\right)=\left(0,2^{t+1}-1\right)$. The existence of this sum-preserving fixed-point-free involution proves that every positive integer $n$ can be written in the form $a+\bar{a}$, where $a \in A_{1}$ and $\bar{a} \in \bar{A}_{1}$, in an even number of ways. A similar proof can be given for $m=2$, and presumably for any $m$, but quickly grows tedious. We now give an algebraic proof that does not depend on $m$.

Theorem 5.1. Let $m \geq 1$. The reciprocal of the set $A_{m}:=\{0\} \cup\left\{2^{m n}: n \in \mathbb{N}\right\}$ is the set

$$
\bar{A}_{m}:=\left\{-1+\sum_{i=0}^{m-1} x_{i} 2^{i+m n_{i}}: x_{i} \in\{0,1\}, \vec{x} \neq \overrightarrow{0}, n_{i} \in \mathbb{N}\right\} .
$$

In particular, both $\delta\left(A_{m}, n\right)$ and $\delta\left(\bar{A}_{m}, n\right)$ are $O_{m}\left(\frac{\log n}{n}\right)$.

Proof. Set $\mathcal{F}(q)=\sum_{n \geq 0} q^{2^{m n}}$. By the children's binomial theorem $\mathcal{F}\left(q^{2}\right)=\mathcal{F}(q)^{2}$, and consequently by induction we see that $\mathcal{F}\left(q^{2^{m}}\right)=\mathcal{F}(q)^{2^{m}}$.

Now, by the definition of $\mathcal{F}, \mathcal{F}\left(q^{2^{m}}\right)=\mathcal{F}(q)+q$ and so

$$
\begin{aligned}
q & =\mathcal{F}(q)^{2^{m}}+\mathcal{F}(q) \\
& =(1+\mathcal{F}(q))\left(\mathcal{F}(q)+\mathcal{F}(q)^{2}+\mathcal{F}(q)^{3}+\cdots+\mathcal{F}(q)^{2^{m}-1}\right) \\
& =(1+\mathcal{F}(q))\left(1+\prod_{i=0}^{m-1}\left(1+\mathcal{F}(q)^{2^{i}}\right)\right) \\
& =(1+\mathcal{F}(q))\left(1+\prod_{i=0}^{m-1}\left(1+\mathcal{F}\left(q^{2^{i}}\right)\right)\right)
\end{aligned}
$$


The series $1+\mathcal{F}(q)$ is the generating function of $\{0\} \cup\left\{2^{m n}: n \in \mathbb{N}\right\}$, and $1+\prod_{i=0}^{m-1}\left(1+\mathcal{F}\left(q^{2^{i}}\right)\right)$ is the generating function of $\left\{\sum_{i=0}^{m-1} x_{i} 2^{i+m n_{i}}: x_{i} \in\{0,1\}, \vec{x} \neq \overrightarrow{0}, n_{i} \in \mathbb{N}\right\}$, so this identity is equivalent to the theorem.

The reader may be interested to note that the reciprocal of the extremely thick set $\mathbb{N} \backslash\left\{2^{n}: n \in\right.$ $\mathbb{N}\}$ is the thin set $\{0,3\} \cup\left\{2^{n}-1,2^{n}-3: n \geq 3\right\}$, whereas the reciprocal of $\mathbb{N} \backslash\left\{4^{n}: n \in \mathbb{N}\right\}$ appears to have density $1 / 2$.

Our next theorem shows that the examples given by Theorem 5.1 are extremal. It is impossible for a set and its reciprocal to both grow sub-logarithmically. This result was suggested to us by Ernest Croot [personal communication].

Theorem 5.2. Let $F, \bar{F}$ be reciprocals (not both $\{0\}$ ), and suppose that $r$ is the least positive integer in $F \cup \bar{F}$. Then

$$
|F \cap[0, n]|+|\bar{F} \cap[0, n]| \geq 2+\left\lfloor\log _{2}(n / r)\right\rfloor .
$$

Proof. First, note that $r \in F \cap \bar{F}$. Let $N \geq r$, so that neither $F \cap[1, N)$ nor $\bar{F} \cap[1, N)$ is empty, and let $m, \bar{m}$ be the largest elements of those sets. Since $q^{m+\bar{m}}$ occurs in the product $\mathcal{F} \overline{\mathcal{F}}$ at least once, it must occur at least twice. Since $N \leq m+\bar{m}<2 N$, we see that

$$
|F \cap[N, 2 N)|+|\bar{F} \cap[N, 2 N)| \geq 1 .
$$

Straightforward counting concludes the proof, since $F \cup \bar{F}$ contains 0 twice, and must intersect each of the intervals $[r, 2 r),\left[2 r, 2^{2} r\right),\left[2^{2} r, 2^{3} r\right), \ldots$.

\section{Theta functions}

Every quadratic that takes integers to integers can be written in the form $c_{0}+c_{1} n+c_{2} \frac{n(n-1)}{2}$ with $c_{i} \in \mathbb{Z}$. We wish to study the ranges of such quadratics, but we only wish to consider sets that contain 0 ; without loss of generality we may take $c_{0}=0$. Thus, we set

$$
\Theta\left(c_{1}, c_{2}\right):=\left\{c_{1} n+c_{2} \frac{n(n-1)}{2}: n \in \mathbb{Z}\right\}
$$

Moreover, we are only interested in those sets that consist of nonnegative integers, so we may assume that $c_{2} \geq c_{1} \geq 0$. And since $\Theta\left(c_{1}, c_{2}\right)=\Theta\left(c_{2}-c_{1}, c_{2}\right)$ we may also assume that $c_{2} \geq 2 c_{1}$. Finally, we are only interested in those sets whose gcd is 1 : we can assume that $\operatorname{gcd}\left(c_{1}, c_{2}\right)=1$. The only set with $c_{1}=0$ not excluded is $\Theta(0,1)=\left\{\left(\begin{array}{l}n \\ 2\end{array}\right): n \geq 1\right\}$, and the only set with $c_{2}=2 c_{1}$ that is not excluded is $\Theta(1,2)=\left\{n^{2}: n \geq 0\right\}$. Otherwise, we have $c_{2}>2 c_{1}>0$, and $\operatorname{gcd}\left(c_{1}, c_{2}\right)=1$.

In Figure 7 we give the number of elements in the reciprocal of $\Theta\left(c_{1}, c_{2}\right)$ (with $c_{2} \leq 18$ ) that are at most $10^{5}$. We note that none of the entries of this table are larger than 50450, and the entries that are less than 49750 are exactly those with $c_{2} \equiv 2(\bmod 4)$. This computation partially justifies Conjecture 1.1

There is another property of $\Theta\left(c_{1}, c_{2}\right)$ that happens exactly when $c_{2} \equiv 2(\bmod 4)$ : the set $\Theta\left(c_{1}, c_{2}\right)$ is not uniformly distributed modulo 4 .

Proposition 6.1. Let $\operatorname{gcd}\left(c_{1}, c_{2}\right)=1$. The set $\Theta\left(c_{1}, c_{2}\right)$ is uniformly distributed modulo every power of 2 if and only if $c_{2} \neq \equiv 2(\bmod 4)$. 


\begin{tabular}{|c|c|c|c|c|c|c|c|c|c|}
\hline & & & & $c$ & & & & & \\
\hline & 0 & 1 & 2 & 3 & 4 & 5 & 6 & 7 & 8 \\
\hline$\overline{\overline{1}}$ & 50162 & & & & & & & & \\
\hline 2 & & 17317 & & & & & & & \\
\hline 3 & & 50201 & & & & & & & \\
\hline 4 & & 50162 & & & & & & & \\
\hline 5 & & 50265 & 49994 & & & & & & \\
\hline 6 & & 17814 & & & & & & & \\
\hline 7 & & 50062 & 50187 & 50449 & & & & & \\
\hline 8 & & 50042 & & 49944 & & & & & \\
\hline$c_{2}$ & & 50214 & 49827 & & 50023 & & & & \\
\hline 10 & & 34009 & & 36084 & & & & & \\
\hline 11 & & 49918 & 50181 & 49918 & 49943 & 49856 & & & \\
\hline 12 & & 49869 & & & & 50254 & & & \\
\hline 13 & & 50089 & 49752 & 49988 & 49992 & 50295 & 49912 & & \\
\hline 14 & & 40981 & & 41776 & & 39062 & & & \\
\hline 15 & & 50004 & 50195 & & 49949 & & & 49900 & \\
\hline 16 & & 50001 & & 49924 & & 49996 & & 50090 & \\
\hline 17 & & 50198 & 49921 & 49932 & 50052 & 50114 & 49826 & 49818 & 49816 \\
\hline 18 & & 48224 & & & & 44500 & & 43772 & \\
\hline
\end{tabular}

Figure 7: The number of elements $\leq 100000$ in the reciprocal of $\Theta\left(c_{1}, c_{2}\right)$

Proof. First, suppose that $c_{1}=2 k+1$ and $c_{2}=4 \ell+2$. Set

$$
f(n):=c_{1} n+c_{2} \frac{n(n-1)}{2}=(2 \ell+1) n^{2}+2(k-\ell) n .
$$

If $k$ and $\ell$ have the same parity, then $f(n) \equiv(2 \ell+1) n^{2}(\bmod 4)$, and since $n^{2}$ takes only two values modulo 4 , the set is not uniformly distributed modulo 4 . If $k$ and $\ell$ have different parity, then

$$
(2 \ell+1) n^{2}+2(k-\ell) n \equiv(2 \ell+1) n^{2}+2 n \quad(\bmod 4)
$$

only takes on the values 0,3 modulo 4 . Thus, if $c_{2} \equiv 2(\bmod 4)$, then $\Theta\left(c_{1}, c_{2}\right)$ is not uniformly distributed modulo 4.

Now suppose that $c_{2}=4 \ell$, and since $\operatorname{gcd}\left(c_{1}, c_{2}\right)=1$, we know that $c_{1}$ is odd. We have

$$
f(n):=c_{1} n+c_{2} \frac{n(n-1)}{2}=2 \ell n^{2}+\left(c_{1}-2 \ell\right) n \equiv n \quad(\bmod 2) .
$$

The formal derivative of $f(n)$ is $4 \ell n+c_{1}-2 \ell \not \equiv 0(\bmod 2)$. By Hensel's Lemma ${ }^{\mathrm{f}}$, the range of the polynomial $f(n)$ hits every congruence class modulo every power of 2 . Since for every $j, f(n)$ is periodic modulo $2^{j}$ with period $2^{j}$, we see that it is uniformly distributed modulo $2^{j}$.

Now suppose that $c_{2}=2 \ell+1$ is odd. Set

$$
\begin{aligned}
G & :=\left\{(2 \ell+1) m(2 m-1)+c_{1}(2 m): m \in \mathbb{Z}\right\} \\
H & :=\left\{(2 \ell+1)(2 m+1) m+c_{1}(2 m+1): m \in \mathbb{Z}\right\}
\end{aligned}
$$

\footnotetext{
${ }^{\mathrm{f} H e n s e l ' s ~ L e m m a: ~ I f ~} f(n)$ is a polynomial with integer coefficients, and the two congruences $f(n) \equiv a$ $(\bmod p), f^{\prime}(n) \not \equiv 0(\bmod p)$ have a simultaneous solution, then $f(n) \equiv a$ has a unique solution modulo every power of the prime $p$.
} 
so that $\Theta\left(c_{1}, 2 \ell+1\right)=G \cup H$. The set $G$ is the range of $g(m):=f(2 m)=(2 \ell+1) m(2 m-$ 1) $+2 c_{1} m \equiv m(\bmod 2)$, which has derivative $g^{\prime}(m) \equiv 1(\bmod 2)$, and the set $H$ is the range of $h(m):=(2 \ell+1)(2 m+1) m+c_{1}(2 m+1) \equiv m+c_{1}(\bmod 2)$, which has derivative $h^{\prime}(m) \equiv 1$ (mod 2). Thus, by Hensel's Lemma, both $G$ and $H$ exhaust every congruence class modulo $2^{j}$, and by periodicity of $g(m)$ and $h(m)$ are therefore uniformly distributed modulo $2^{j}$.

\subsection{The squares}

Let $\mathcal{S}(q)=\sum_{n=0}^{\infty} q^{n^{2}}$, and $S=\{0,1,4,9,16,25, \ldots\}$. Figure 2 shows $\delta(\bar{S}, x)$ for two ranges of $x$. On the small scale, we see that the relative density behaves irregularly, with many small increases and decreases. On the larger scale, we see that the relative density seems to decrease inexorably.

We characterize completely the values of $\bar{S}$ in the residue classes $0,1,2(\bmod 4)$.

Let $\nu_{p}(n)$ be the integer such that $p^{\nu_{p}(n)} \mid n$ and $p^{\nu_{p}(n)+1} \nmid n$, so that

$$
n=\prod_{p \text { prime }} p^{\nu_{p}(n)}
$$

for every $n$. Let $r_{2}(n)$ be the number of representations of $n$ in the form $y^{2}+z^{2}$, where $y$ and $z$ are integers.

Theorem 6.2. Let $n \in \mathbb{N}$. If $n$ is even, then $n \in \bar{S}$ if and only if $n$ is twice a square. If $n \equiv 1$ $(\bmod 4)$ is not a square, then $n \in \bar{S}$ if and only if $\nu_{p}(n)$ is even for every prime $p$ except one, and that prime $p$ and $\nu_{p}(n)$ are both congruent to 1 modulo 4 . If $n \equiv 1(\bmod 4)$ is a square, then $n \in \bar{S}$ if and only if $\nu_{p}(n) \equiv 2(\bmod 4)$ for an even number of primes $p \equiv 1(\bmod 4)$.

We will need the following lemmas. The first expresses $\bar{s}_{n}$ in terms of the number of representations of $n$ by a particular (depending on $n$ ) quadratic form. The second is quoted without proof from [12, and gives a formula for $r_{2}(n)$.

Lemma 6.3. Let $n \in \mathbb{N}$, and let $j \in \mathbb{N}$ satisfy $n \equiv 2^{j}-1\left(\bmod 2^{j+1}\right)$. Then $\bar{s}_{n}=1$ if and only if

$$
\#\left\{\left(k_{0}, \ldots, k_{j-1}, k_{j+1}\right): k_{i} \in \mathbb{N}, n=2^{j+1} k_{j+1}^{2}+\sum_{i=0}^{j-1} 2^{i} k_{i}^{2}\right\}
$$

is odd.

Proof. By Lemma $2.2(i i), \bar{s}_{n}=1$ exactly if there are an odd number of tuples $\left(k_{0}, k_{1}, \ldots\right)$ with weight

$$
n=k_{0}^{2}+2 k_{1}^{2}+4 k_{2}^{2}+8 k_{3}^{2}+\cdots .
$$

Let $w(n)$ be the number of such tuples. We give a weight-preserving involution $\mu$ of such tuples, and deduce the lemma from

$$
w(n) \equiv \#(\text { fixed points of } \mu \text { with weight } n) \quad(\bmod 2) .
$$

Since $n \not \equiv 2^{i}-1\left(\bmod 2^{i+1}\right)$ for $0 \leq i<j$, reducing (8) modulo $2,4, \ldots, 2^{j}$ successively tells us that $k_{0}, k_{1}, \ldots, k_{j-1}$ are odd, while $n \equiv 2^{j}-1\left(\bmod 2^{j+1}\right)$ tells us that $k_{j}$ is even. Now define $J$ to be the least integer with the two properties: $J \geq j+2$; and $2 k_{J} \neq k_{j}$.

We define

$$
\mu\left(k_{0}, k_{1}, k_{2}, \ldots\right)=\left(k_{0}, k_{1}, \ldots, k_{j-1}, 2 k_{J}, k_{j+1}, k_{J}, k_{J}, \ldots, k_{J}, k_{j} / 2, k_{J+1}, k_{J+2}, \ldots\right),
$$


where $k_{J}$ is repeated $J-j-2$ times. That this is a weight-preserving involution is a routine calculation.

The fixed points of $\mu$ are those tuples with $0=k_{j}=k_{j+2}=k_{j+3}=\cdots$. In other words, there is a fixed point for each solution to

$$
n=k_{0}^{2}+2 k_{1}^{2}+\cdots+2^{j-1} k_{j-1}^{2}+2^{j+1} k_{j+1}^{2} .
$$

Lemma 6.4 ([12, Theorem 3.22]). If $\nu_{p}(n)$ is odd for any prime $p$ congruent to 3 (modulo 4), then $r_{2}(n)=0$. Otherwise, $r_{2}(n)=4 \prod_{p}\left(\nu_{p}(n)+1\right)$, where the product extends over all primes congruent to 1 (modulo 4).

Proof of Theorem 6.2. If $n$ is even, then $n \equiv 2^{0}-1\left(\bmod 2^{0+1}\right)$, so we can apply Lemma 6.3 with $j=0$ to arrive at $\bar{s}_{n}=1$ if and only if $n$ has an odd number of representations of the form $2 k_{1}^{2}$ (with $k_{1} \geq 0$ ). Clearly there cannot be more than one such representation, and there is one exactly if $n$ is twice a perfect square.

If $n \equiv 1(\bmod 4)$, then we may apply Lemma 6.3 with $j=1$ to arrive at $\bar{s}_{n}=1$ if and only if $n$ has an odd number of representations of the form $k_{0}^{2}+4 k_{2}^{2}$ (with $k_{0}$ and $k_{2}$ nonnegative).

We assume for now that $n$ is not a square. Since $n$ is odd, there are no such representations with $k_{0}=0$, and since $n$ is not a square, there are no such representations with $k_{2}=0$. Thus, every such representation $k_{0}^{2}+4 k_{2}^{2}$ gives rise to 8 representations $\left\{\left( \pm k_{0}\right)^{2}+\left( \pm 2 k_{2}\right)^{2},\left( \pm 2 k_{2}\right)^{2}+\left( \pm k_{0}\right)^{2}\right\}$ of $n$ in the form $y^{2}+z^{2}$. Moreover, any solution to $n=y^{2}+z^{2}$ must have one of $y$ or $z$ even and the other odd since $n$ is odd, and $y \neq z$ since $n$ is odd. Since $n$ is not a square, neither $y$ nor $z$ is zero. Every representation $(y, z)$ occurs as one of a family of 8 such representations, and one of these has $n=y^{2}+z^{2}=y^{2}+4(z / 2)^{2}$ with $y>0$ and $z>0$. Thus, $\bar{s}_{n}=1$ if and only if $r_{2}(n) / 8$ is odd.

By Lemma 6.4 $r_{2}(n) / 8=0$ if $\nu_{p}(n)$ is odd for any prime $p$ congruent to 3 modulo 4 . Otherwise, $r_{2}(n) / 8=\frac{1}{2} \prod_{p}\left(\nu_{p}(n)+1\right)$, where the product extends over those primes that are congruent to 1 modulo 4 (in the remainder of this paragraph, $p$ is always 1 modulo 4 ). First, note that $\nu_{p}(n)$ is odd for some prime $p$ since $n$ is not a square. If some $\nu_{p}(n)$ is 3 modulo 4 for some $p$, then $r_{2}(n) / 8$ is even, and similarly if $\nu_{p}(n)$ is 1 modulo 4 for two primes $p$. Thus, $r_{2}(n) / 8$ is odd precisely if $\nu_{p}(n)$ is odd for exactly one prime, and both that prime and $\nu_{p}(n)$ are 1 modulo 4 .

Now we assume that $n \equiv 1(\bmod 4)$ is a square, say $n=x^{2}$. Then, as above, most representations of $n$ in the form $k_{0}^{2}+4 k_{2}^{2}$ correspond to 8 representations of $n$ in the form $y^{2}+z^{2}$, but the representation $n=x^{2}+4 \cdot 0^{2}$ only corresponds to 4 representations in the form $y^{2}+z^{2}$. Since $n$ is a square, we know that $\nu_{p}(n)$ is even for every prime $p$. Thus, $\bar{s}_{n}=1$ if and only if

$$
\frac{r_{2}(n)-4}{8}+1
$$

is odd. Using the formula from Lemma 6.4 this happens exactly if $1 \equiv \prod_{p}\left(\nu_{p}(n)+1\right)(\bmod 4)$, where the product extends over primes that are 1 modulo 4 . This, in turn, happens exactly when $\nu_{p}(n) \equiv 2(\bmod 4)$ for an even number of primes $p \equiv 1(\bmod 4)$.

We suspect that $\delta(\bar{S})=0$ and that this may follow from the theory of modular forms, but again, this is outside the scope of this paper. We emphasize in Corollary 6.5 that our characterization of $\bar{S}$ is consistent with Conjecture 1.1

Corollary 6.5. The set $\{n \in \mathbb{N}: n \in \bar{S}, n \not \equiv 3(\bmod 4)\}$ has zero density.

Proof. By Theorem 6.2] the set $\bar{S}$ clearly has no density in $0 \bmod 2$. We will use the description given in Theorem 6.2 to show that $\bar{S}$ also has zero density in $1 \bmod 4$. 
By the Wiener-Ikehara Theorem (see [4 Section 7.2]), we have for any set $A$ of positive integers

$$
\lim _{n \rightarrow \infty} \delta(A, n)=\lim _{s \rightarrow 1+}(s-1) \sum_{a \in A} a^{-s} .
$$

Set $A=\left\{n^{2} p: 1 \leq n \in \mathbb{N}, p\right.$ prime $\}$, and observe that $\delta(A)=0$ since

$$
\begin{aligned}
\delta(A) & \leq \lim _{s \rightarrow 1+}(s-1) \sum_{a \in A} a^{-s} \\
& =\lim _{s \rightarrow 1+}(s-1)\left(\prod_{p \text { prime }}\left(1-p^{-2 s}\right)^{-1}\right)\left(\sum_{p \text { prime }} p^{-s}\right) \\
& =\lim _{s \rightarrow 1+}(s-1) \zeta(2 s)\left(\sum_{p \text { prime }} p^{-s}\right) \\
& =\zeta(2) \delta(\text { primes })=0
\end{aligned}
$$

Note that the subset of $\bar{S}$ whose elements are even has density 0 , and the subset whose elements are congruent to 1 modulo 4 is (except for some squares) contained in $A$. Thus

$$
\delta(\{n \in \bar{S}: n \not \equiv 3 \quad(\bmod 4)\}) \leq \delta(\text { squares })+\delta(A)=0 .
$$

\section{Prouhet-Thue-Morse numbers}

Set $t_{n}=1$ if the binary expansion of $n$ contains an even number of " 1 "s, and set $t_{n}=0$ otherwise. The set $T:=\left\{n: t_{n}=1\right\}=\{0,3,5,6,9, \ldots\}$ is called the Prouhet-Thue-Morse sequence. This sequence frequently arises because it simultaneously has enough structure to analyze, and enough "random-like" behavior to be interesting. The survey [3] details four of the occasions that the sequence has been independently rediscovered: first in number theory (Prouhet), then combinatorics (Thue), then in differential geometry (Morse), and finally chess grandmaster Max Euwe rediscovered it to demonstrate that the rules then in use did not imply that chess is a finite game.

For every $n \in \mathbb{N}, 2 n \in T$ if and only if $2 n+1 \notin T$; thus $\mathcal{T}(q):=\sum_{n=0}^{\infty} t_{n} q^{n}$ has $\delta(\mathcal{T})=1 / 2$. The sequence $t_{0}, t_{1}, \ldots$ is not eventually periodic (in fact, the real number with binary expansion $0 . t_{0} t_{1} t_{2} \cdots$ is transcendental [2,7]), so $\overline{\mathcal{T}}$ is not a polynomial. A counting argument [3] reveals the interesting identity:

$$
(1+q)^{3} \mathcal{T}(q)^{2}+(1+q)^{2} \mathcal{T}(q)=q
$$

Multiplying by $\overline{\mathcal{T}}(q)$ yields $q \overline{\mathcal{T}}(q)=\left(1+q+q^{2}+q^{3}\right) \mathcal{T}(q)+1+q^{2}$, whence for $n \geq 2$

$$
\bar{t}_{n}=t_{n+1}+t_{n}+t_{n-1}+t_{n-2} .
$$

This leads reasonably directly (albeit with the modest labor involved in deriving (92) to a proof of Theorem 7.1 Instead, we give a proof which does not rely on the special form of the functional equation (91), and so is more representative of the process of finding reciprocals.

Theorem 7.1. The reciprocal of the set $T$ of Prouhet-Thue-Morse numbers is

$$
\bar{T}=\{0\} \cup\{4 k \pm 1 \text { : the binary expansion of } k \geq 1 \text { ends in an even number of " } 1 \text { " } s\} \text {. }
$$

Consequently, $\delta(\overline{\mathcal{T}})=1 / 3$. 
If (the binary expansion of) $k$ ends in an even number of "1"s, then $4 k+1$ ends with a string $10^{2 k+1} 1$ (a "1" followed by an odd number of "0"s followed by a single "1"), while $4 k-1$ ends with a string $01^{2 k}$ (a "0" followed by a positive even number of "1"s).

Proof. By Lemma 2.1] $\overline{\mathcal{T}}(q)=\sum_{n=0}^{\infty} r(n) q^{n}$, where $r(n)$ is the number of ways to write $n$ as

$$
n=s_{0}+2 s_{1}+4 s_{2}+8 s_{3}+\cdots+2^{k} s_{k}+\cdots
$$

where the $s_{k}$ are Prouhet-Thue-Morse numbers. We will build an involution $\tau$ on the set of such representations, and $r(n)$ will have the same parity as the number of fixed points of $\tau$.

By a tuple, we mean an infinite list of Prouhet-Thue-Morse numbers which is 0 from some point on. The weight of a tuple $\left(s_{0}, s_{1}, \ldots\right)$ is $\sum_{n=0}^{\infty} s_{n} 2^{n}$.

We now give the weight-preserving permutation $\tau$ of the set of tuples which is actually an involution. The permutation $\tau$ has an odd number of fixed points with weight $n>0$ if and only if the binary expansion of $n$ ends with a string $10^{2 k+1} 1$ (a " 1 " followed by an odd number of "0"s followed by a single "1") or ends with a string $01^{2 k}$ (a "0" followed by a positive even number of "1"s). These are exactly the numbers of the form $4 k \pm 1$, where the binary expansion of $k$ ends in an even number of "0" s, and this will conclude the proof.

Defining the permutation $\tau$ : Suppose that $s_{0}$ is even. If $s_{0} \neq 2 s_{1}$, then set

$$
\tau\left(s_{0}, s_{1}, s_{2}, \ldots\right):=\left(2 s_{1}, s_{0} / 2, s_{2}, s_{3}, \ldots\right) .
$$

If $s_{0}=2 s_{1}$, then let $i$ be minimal with $s_{1} \neq s_{i}$, and set

$$
\tau\left(s_{0}, s_{1}, s_{2}, \ldots\right):=\left(2 s_{i}, s_{i}, s_{i}, \ldots, s_{i}, s_{1}, s_{i+1}, s_{i+2}, \ldots\right),
$$

where $s_{i}$ is repeated $i-1$ times. The only fixed point with $s_{0}$ even is $(0,0, \ldots)$ with weight 0 .

Now suppose that $s_{0} \equiv 3(\bmod 4)$. Since $\left(s_{0}-3\right) / 2$ is even, we can define $v_{0}, v_{2}, v_{3}, \ldots$ by

$$
\left(v_{0}, v_{2}, v_{3}, \ldots\right):=\tau\left(\left(s_{0}-3\right) / 2, s_{2}, s_{3}, \ldots\right),
$$

where the action of $\tau$ has already been defined above. Note that $v_{1}$ is not defined, and that $s_{1}$ has not been used. We now set

$$
\tau\left(s_{0}, s_{1}, s_{2}, \ldots\right):=\left(2 v_{0}+3, s_{1}, v_{2}, v_{3}, \ldots\right) .
$$

The only fixed points with $s_{0} \equiv 3(\bmod 4)$ are the tuples of the form $\left(3, s_{1}, 0,0, \ldots\right)$, where $s_{1}$ is a Prouhet-Thue-Morse number. These fixed points have weight $3+2 s_{1}$.

Now suppose that $s_{0} \equiv 1(\bmod 4)$. If there exists an $L$ such that $s_{i}=\left(s_{0}+1\right) / 2$ for $0<i \leq L$ and $s_{i}=0$ for $i>L$, then we let $\tau$ fix the tuple. These will be the only fixed points of $\tau$ with $s_{0} \equiv 1$ $(\bmod 4)$, and will have weight $2^{L} s_{0}+2^{L}-1$. Otherwise, if any $s_{i}$ is even (except for the tail of zeros in the tuple $\left.\left(s_{0}, s_{1}, s_{2}, \ldots\right)\right)$, then let $K:=\min \left\{i: s_{i}\right.$ even $\}$, and set

$$
\tau\left(s_{0}, s_{1}, s_{2}, \ldots\right):=\left(s_{0}, s_{1}, s_{2}, \ldots, s_{K-1}, \tau\left(s_{K}, s_{K+1}, s_{K+2}, \ldots\right)\right) .
$$

If on the other hand all $s_{i}$ are odd (except for the ending string of zeros), then define $v_{0}, v_{1}, v_{2}, \ldots$ by

$$
\left(v_{0}, v_{1}, v_{2}, \ldots\right):=\tau\left(s_{0}+1, s_{1}, s_{2}, s_{3}, \ldots\right)
$$

and set

$$
\tau\left(s_{0}, s_{1}, s_{2}, \ldots\right):=\left(v_{0}-1, v_{1}, v_{2}, \ldots\right) .
$$

That $\tau$ is an involution with precisely the claimed fixed points is simply a matter of checking the various cases; we cheerfully leave this important tedium to the reader. 
Analysis of $\tau$ 's fixed points with weight $n$ : Suppose that $n$ is even. By parity considerations, we see that all tuples $\left(s_{0}, s_{1}, \ldots\right)$ with weight $\sum_{i=0}^{\infty} s_{i} 2^{i}=n$ have $s_{0}$ even. Since the only fixed point with $s_{0}$ even is $(0,0, \ldots)$, we see that $r(0)=1$ and $r(n)$ is even for all even $n>0$. From this point on we assume that $n$ is odd.

Suppose that $n \equiv 1(\bmod 4)$, and $\left(s_{0}, s_{1}, \ldots\right)$ is a fixed point of $\tau$ with weight $n$. Since $n$ is odd, $s_{0}$ is either 1 or 3 modulo 4 . If $s_{0} \equiv 1(\bmod 4)$, then $s_{1} \equiv 1(\bmod 2)$, and such a tuple can be fixed by $\tau$ only if $n=s_{0}$, and $n$ is a Prouhet-Thue-Morse number. If $s_{0} \equiv 3(\bmod 4)$, then $s_{1} \equiv 1(\bmod 2)$, and such a tuple can be fixed by $\tau$ only if $n=3+2 s_{1}$, i.e., if $(n-3) / 2$ is a Prouhet-Thue-Morse number (and in this case there is exactly one such tuple). Thus $\tau$ has either 0,1 , or 2 fixed points, and we care about when it has an odd number of fixed points. Since $n \equiv 1$ $(\bmod 4)$, the binary expansion of $n$ can be written as $\left(\mathrm{x} 10^{k} 1\right)_{2}$ for some binary string $\mathrm{x}$ and positive integer $k$. We see that the binary expansion of $(n-3) / 2$ is $\left(\mathrm{x}_{0} 1^{k}\right)_{2}$. Thus, if $k$ is even, then either both $n$ and $(n-3) / 2$ are Prouhet-Thue-Morse numbers or neither is. If $k$ is odd, then exactly one of $n$ and $(n-3) / 2$ are Prouhet-Thue-Morse numbers. Hence, $\tau$ has an odd number of fixed points exactly if the binary expansion of $n$ ends in $10^{k} 1$, with $k$ an odd number.

Now suppose that $n \equiv 3(\bmod 4)$, and $\left(s_{0}, s_{1}, \ldots\right)$ is a fixed point of $\tau$ with weight $n$. Since $n$ is odd, $s_{0}$ is either 1 or 3 modulo 4 . If $s_{0} \equiv 1(\bmod 4)$, then $s_{1} \equiv 1(\bmod 2)$, and such a tuple can be fixed by $\tau$ only if $s_{i}=\left(s_{0}+1\right) / 2$ for all $0<i \leq L$ and $s_{i}=0$ for $i>L$. In this case, $n=2^{L} s_{0}+\left(2^{L}-1\right)$. Since $s_{0} \equiv 1(\bmod 4)$, this implies that the binary expansion of $n$ ends with $L+1$ "1"s (in particular, at most one value of $L$ can lead to such a fixed point). Moreover, $2^{L} s_{0}+\left(2^{L}-1\right)$ is a Prouhet-Thue-Morse number if and only if $L$ is even. If $s_{0} \equiv 3(\bmod 4)$, then $s_{1} \equiv 0(\bmod 2)$, and such a tuple is fixed if and only if it is of the form $\left(3, s_{1}, 0,0, \ldots\right)$. This can happen exactly if $(n-3) / 2$ is a Prouhet-Thue-Morse number.

Suppose that $n$ is a Prouhet-Thue-Morse number. If the binary expansion of $n$ ends in exactly $2 k>0$ " 1 "s, then $(3,(n-3) / 2,0,0, \ldots)$ is the only fixed point of $\tau$. If the binary expansion of $n$ ends in $2 k+1>0$ " 1 "s, then both $(3,(n-3) / 2,0,0, \ldots)$ and

$$
\left(\frac{n-2^{2 k}+1}{2^{2 k}}, \frac{n+1}{2^{2 k+1}}, \frac{n+1}{2^{2 k+1}}, \ldots, 0,0, \ldots\right)
$$

(the term $(n+1) / 2^{2 k+1}$ is repeated $2 k$ times) are fixed points.

Now suppose that $n$ is not a Prouhet-Thue-Morse number. If the binary expansion of $n$ ends in exactly $2 k>0$ "1"s, then

$$
\left(\frac{n-2^{2 k-1}+1}{2^{2 k-1}}, \frac{n+1}{2^{2 k}}, \frac{n+1}{2^{2 k}}, \ldots, 0,0, \ldots\right)
$$

(the term $(n+1) / 2^{2 k}$ is repeated $2 k-1$ times) is the only fixed point. If the binary expansion of $n$ ends in $2 k+1>0$ " 1 "s, then there are no fixed points.

\section{References}

[1] Scott Ahlgren, Distribution of parity of the partition function in arithmetic progressions, Indag. Math. (N.S.) 10 (1999), 173-181. MR 1816213

[2] J.-P. Allouche, J. L. Davison, M. Queffélec, and L. Q. Zamboni, Transcendence of Sturmian or morphic continued fractions, J. Number Theory 91 (2001), 39-66. MR 1869317

[3] Jean-Paul Allouche and Jeffrey Shallit, The ubiquitous Prouhet-Thue-Morse sequence, Sequences and Their Applications (Singapore, 1998), Springer Ser. Discrete Math. Theor. Comput. Sci., Springer, London, 1999, pp. 1-16. MR 1843077 
[4] Paul T. Bateman and Harold G. Diamond, Analytic number theory, World Scientific Publishing Co. Ptd. Ltd., Hackensack, NJ, 2004, ISBN 981-256-080-7, An introductory course. MR 2111739

[5] Bruce C. Berndt, Ae Ja Yee, and Alexandru Zaharescu, New theorems on the parity of partition functions, J. Reine Angew. Math. 566 (2004), 91-109. MR 2039324

[6] _ On the parity of partition functions, Internat. J. Math. 14 (2003), 437-459. MR 1984662

[7] Michel Dekking, Transcendance du nombre de Thue-Morse, C. R. Acad. Sci. Paris Sér. A-B 285 (1977), A157A160. MR 0457363 (French, with English summary)

[8] Harold Fredricksen, A survey of full length nonlinear shift register cycle algorithms, SIAM Rev. 24 (1982), 195221. MR 652466

[9] Solomon W. Golomb, Shift register sequences, With portions co-authored by Lloyd R. Welch, Richard M. Goldstein, and Alfred W. Hales, Holden-Day Inc., San Francisco, Calif., 1967. MR 0242575

[10] E. E. Kummer, Über die Ergänzungssätze zu den allgemeinen Reciprocitätsgesetzen, J. Reine Angew. Math. 44 (1852), 93-146.

[11] J.-L. Nicolas, I. Z. Ruzsa, and A. Sárközy, On the parity of additive representation functions, J. Number Theory 73 (1998), 292-317, With an appendix in French by J.-P. Serre. MR 1657968

[12] Ivan Niven, Herbert S. Zuckerman, and Hugh L. Montgomery, An introduction to the theory of numbers, 5th ed., John Wiley \& Sons Inc., New York, 1991, ISBN 0-471-62546-9. MR 1083765

[13] Thomas R. Parkin and Daniel Shanks, On the distribution of parity in the partition function, Math. Comp. 21 (1967), 466-480. MR 0227126

[14] David Williams, Probability with martingales, Cambridge Mathematical Textbooks, Cambridge University Press, Cambridge, 1991, ISBN 0-521-40455-X, 0-521-40605-6. MR 1155402 